\title{
CAYLEY-BACHARACH SCHEMES AND THEIR CANONICAL MODULES
}

\author{
ANTHONY V. GERAMITA, MARTIN KREUZER AND LORENZO ROBBIANO
}

\begin{abstract}
A set of $s$ points in $\mathbb{P}^{d}$ is called a Cayley-Bacharach scheme (CBscheme), if every subset of $s-1$ points has the same Hilbert function. We investigate the consequences of this "weak uniformity." The main result characterizes CB-schemes in terms of the structure of the canonical module of their projective coordinate ring. From this we get that the Hilbert function of a CB-scheme $X$ has to satisfy growth conditions which are only slightly weaker than the ones given by Harris and Eisenbud for points with the uniform position property. We also characterize CB-schemes in terms of the conductor of the projective coordinate ring in its integral closure and in terms of the forms of minimal degree passing through a linked set of points. Applications include efficient algorithms for checking whether a given set of points is a CB-scheme, results about generic hyperplane sections of arithmetically Cohen-Macaulay curves and inequalities for the Hilbert functions of Cohen-Macaulay domains.
\end{abstract}

\section{INTRODUCTION}

There are many ways in which the study of finite sets of points in projective spaces has entered into geometric discussions about other objects. One of them is due to Castelnuovo and centers around the study of smooth and irreducible curves.

It is well known that the genus $g$ of a curve in $\mathbb{P}^{2}$ is determined by its degree $d$ via the formula $g=\frac{1}{2}(d-1)(d-2)$. It was also known to geometers of the last century that for curves in $\mathbb{P}^{r}$ this formula represents an extreme among the possibilities for the genus of a curve of degree $d$. One result that Castelnuovo showed in [C] was that if $C$ is not contained in a hyperplane, then $g \leq \chi\left(d-r-\frac{1}{2}(\chi-1)(r-1)\right)$, where $\chi$ is the integer with $\frac{d-1}{r-1}-1 \leq \chi<\frac{d-1}{r-1}$. $\mathrm{He}$ also studied and classified curves whose genera are extremal with respect to this inequality. Castelnuovo's major tool is a careful examination of the postulation of a general hyperplane section of $C$. For a detailed discussion of his method we refer the reader to the manuscript [HE].

More recently, J. Harris has extended the scope of Castelnuovo's approach with his proof of the Uniform Position Lemma in [H]. This lemma asserts that a general hyperplane section (in characteristic 0 ) of an integral curve has the uniform position property, i.e. the property that any two subsets of $X$ with the same cardinality have the same Hilbert function.

Received by the editors January 3, 1991 and, in revised form, April 26, 1991.

1991 Mathematics Subject Classification. Primary 13C13, 14A05; Secondary 13P10, 14M05, $14 \mathrm{M} 99,14 \mathrm{Q} 99$. 
If $X$ is a set of points in $\mathbb{P}^{2}$ with the uniform position property (e.g. a general hyperplane section of an integral curve in $\mathbb{P}^{3}$ ), then it is possible to completely describe all the numerical functions that could be the Hilbert function of $X$. It is also possible to show that every such numerical function actually arises as the Hilbert function of a general hyperplane section of some integral curve in $\mathbb{P}^{3}$. For a discussion of the work that has been done on this subject see [GM].

As soon as we look at general hyperplane sections of curves in $\mathbb{P}^{d}$ for $d \geq 4$, the situation becomes much less clear. Harris and Eisenbud have asked for a characterization of the Hilbert functions of sets of points with the uniform position property, and, as yet, there is no solution to this problem. There have, however, been some partial results. Most notably, Harris and Eisenbud themselves give a growth condition that the Hilbert function of a set of points with the uniform position property has to satisfy (cf. [HE, 3.5]). It is clear that their growth condition is not sufficient to characterize those Hilbert functions, since it fails to do so in $\mathbb{P}^{2}$.

Now we come to a description of our contribution to this discussion. In a nutshell, what we have done is postulated a small piece of the uniform position property and investigated the consequences. More precisely, we say that a set $X$ of $s$ points in $\mathbb{P}^{d}$ is a Cayley-Bacharach scheme (CB-scheme), if every subset of $s-1$ points has the same Hilbert function. The theme of this paper is then to find out how much information about $X$ can be obtained from this "weak uniformity."

One consequence is that every subset $Y$ of $X$ that consists of $s-1$ points has Hilbert function $H_{Y}(n)=\min \left\{H_{X}(n), s-1\right\}$. Therefore we start in $\S 1$ with an investigation of the Hilbert functions of subschemes of degree $s-1$ of $X$. For this we need not restrict ourselves to reduced 0-dimensional schemes $X$ of $\mathbb{P}^{d}$. We find certain functions, called truncators, which define the subschemes of $X$ of degree $s-1$ scheme-theoretically, and which can be used to determine whether those subschemes have the Hilbert function given above. Moreover, the images of those functions in the projective coordinate ring $R$ of $X$ form a $k$-basis of $R_{a_{X}+1}$, where $a_{X}+1$ is the first degree $n$ for which $H_{X}(n)=s$, and they can be used to completely describe the ring structure of $R$ from that degree onwards.

In $\S 2$ we refine the notion of a truncator to include functions of smaller degrees defining subschemes of colength one. For a reduced 0-dimensional scheme $X$ and a point $P \in X$, the smallest degree of such a function is called the degree of $P$ in $X$. Then $X$ is a CB-scheme if all of its points have (maximal) degree $a_{X}+1$. When we look at examples, we immediately see that it is necessary to understand when a subscheme of a CB-scheme is again a CB-scheme. The remainder of $\S 2$ is devoted to giving a few results in that direction.

The central result of this paper is given in $\S 3$. Theorem 3.5 characterizes CB-schemes in terms of the structure of the canonical module $\omega_{R}$ of their projective coordinate ring $R$. More precisely, it says that $X$ is a CB-scheme if and only if the homogeneous component of least degree of $\omega_{R}$ contains a faithful element. From this theorem we get that the Hilbert function of a CB-scheme $X$ has to satisfy growth conditions which are only slightly weaker than the ones given by Harris and Eisenbud for points with the uniform position property. Section 3 concludes with a comparison of our characterization of CB- 
schemes to earlier characterizations that were given in terms of the conductor of $R$ in its integral closure. The link is established by showing that Dedekind's formula for conductor and complementary module always holds for sets of points.

In $\S 4$ we explore another way of interpreting what it means for a set of points to be a CB-scheme. Choose a reduced 0-dimensional complete intersection $Z$ containing $X$ and let $Y$ be the complement of $X$ in $Z$, i.e. $X$ and $Y$ are linked by the complete intersection $Z$. We then characterize CB-schemes $X$ in terms of the forms of minimal degree that vanish on $Y$. One advantage of this characterization is that it leads to efficient algorithms for checking whether a given $X$ is a CB-scheme.

In the final section we apply our results to hyperplane sections of curves. We show that the general hyperplane section of an integral, arithmetically CohenMacaulay curve is a CB-scheme, regardless of the characteristic of the ground field. In contrast to the topological arguments in J. Harris' proof of the Uniform Position Lemma, we use a purely algebraic technique. We also compare the inequalities that we obtain to recent results of R. P. Stanley about the $h$-vector of a Cohen-Macaulay domain and to Rathmann's results about the validity of the Uniform Position Lemma in characteristic $p$. We close with an example that shows that not every hyperplane section of a smooth, arithmetically CohenMacaulay curve has to be a CB-scheme.

Acknowledgments. The authors would like to thank T. Hibi for pointing out how the canonical module of a set of points can be used to get results about its Hilbert function, and E. Kunz for explaining the link between the conductor and the canonical module. The second and third authors are very grateful for the hospitality and support of the Department of Mathematics and Statistics of Queen's University, Kingston, during part of the preparation of this paper. All three authors gratefully acknowledge financial support from the Natural Science and Engineering Research Council of Canada.

\section{TRUnCATORS}

First of all we fix the notation that will be used throughout the paper. We work over an infinite field $k$. By $X$ we denote a 0 -dimensional subscheme of the projective space $\mathbb{P}^{d}$ over $k$. The degree of $X$ as a projective variety is denoted by $s$. It is also the multiplicity of the vertex of the affine cone over $X$. We equip $A:=k\left[X_{0}, \ldots, X_{d}\right]$ with its standard grading $\operatorname{deg}\left(X_{i}\right)=1$ and let $I:=I(X)$ be the homogeneous ideal of $X$ in $A$. Then $R:=A / I$ is the homogeneous coordinate ring of $X$ in $\mathbb{P}^{d}$. To avoid overburdening the notation we will use capital letters to denote elements in $A$ and the corresponding lower case letters to denote their residue classes in $R$. Once and for all, we choose our coordinates in $\mathbb{P}^{d}$ in such a way that $x_{0}$, the image of $X_{0}$ in $R$, is not a zero divisor of $R$.

Given any homogeneous ideal $J$ of $A$, we define the Hilbert function $H(S)$ of the ring $S:=A / J$ by $H(S, n):=\operatorname{dim}_{k} S_{n}=\operatorname{dim}_{k} A_{n}-\operatorname{dim}_{k} J_{n}$ for all $n \in \mathbb{Z}$. Analogously, for a finitely generated, graded $S$-module $M$, we let $H(M, n):=\operatorname{dim}_{k} M_{n}$ for all $n \in \mathbb{Z}$. When $J=I(X)$, we emphasize the relationship of the Hilbert function of $R$ to the scheme $X$ by setting $H_{X}(n):=$ $H(R, n)$ for all $n \in \mathbb{Z}$. 
Remark 1.1. We recall some rules which describe a part of the growth behaviour of the function $H_{X}$ (see e.g. [DGM]). There are integers $\alpha_{X}$ and $a_{X}$ such that

(a) $H_{X}(n)=\operatorname{dim}_{k} A_{n}=\left(\begin{array}{c}n+d \\ d\end{array}\right)$ if and only if $n<\alpha_{X}$,

(b) $H_{X}(n)<H_{X}(n+1)<s$ for $0 \leq n<a_{X}$,

(c) $H_{X}(n)=s$ for $n>a_{X}$.

Given $X$, it is in general difficult to say much about the Hilbert functions of the various subschemes of $X$. This problem was explored in [GMR], where the notion of the truncation of $H_{X}$, as defined below, was discussed. We shall extend some of the results of that paper in this section.

Definition 1.2. The truncation of $H_{X}$, denoted $\operatorname{Trunc}\left(H_{X}\right)$, is defined by

$$
\operatorname{Trunc}\left(H_{X}\right)(n)= \begin{cases}H_{X}(n) & \text { for } n \leq a_{X}, \\ s-1 & \text { for } n>a_{X} .\end{cases}
$$

We say that a homogeneous ideal $J$ of $A$ truncates $H_{X}$, if $J$ contains $I$ and $H(A / J)=\operatorname{Trunc}\left(H_{X}\right)$.

Proposition 1.3. Let $J \subseteq A$ be a homogeneous ideal. The following conditions are equivalent.

(a) $J$ truncates $H_{X}$.

(b) There is a homogeneous polynomial $F \in A$ of degree $a_{X}+1$ such that $J=(I, F)$, and $\operatorname{dim}_{k}\left(J_{n} / I_{n}\right)=1$ for $n>a_{X}$.

Proof. First we show that (a) implies (b). Since $J$ truncates $H_{X}$, we obtain that

$$
\operatorname{dim}_{k} J_{n} / I_{n}=H_{X}(n)-H(A / J, n)= \begin{cases}0 & \text { for } n \leq a_{X}, \\ 1 & \text { for } n>a_{X} .\end{cases}
$$

It follows that there is an $F \in J_{a_{X}+1} \backslash I_{a_{X}+1}$. Since $x_{0}$ is not a zero divisor of $R=A / I$, we have $X_{0}^{i} F \in J_{a_{X}+1+i} \backslash I_{a_{X}+1+i}$ for all $i \geq 0$. Thus $J=(I, F)$.

As for the converse, since $\operatorname{deg} F=a_{X}+1$, we have that $J_{n}=I_{n}$ for $n \leq a_{X}$, and so $H(A / J, n)=H_{X}(n)$ for those $n$. For $n>a_{X}$ we get $H(A / J, n)=$ $\operatorname{dim}_{k}(A / I)_{n}-\operatorname{dim}_{k}(J / I)_{n}=H_{X}(n)-1=s-1$, as we wanted to show.

In general, an ideal $J$ (as above) is not the ideal of the subscheme of $X$ that it defines. It may happen that the irrelevant ideal of $A$ is an associated prime ideal of $J$, i.e. that $J$ is not a saturated ideal. It is important to be able to decide when $J$ is saturated. In the light of Proposition 1.3 and in order to discuss this possibility, we make the following definitions.

Definition 1.4. Let $F \in A$ be homogeneous of degree $a_{X}+1$.

(a) We say that $F$ is a truncator of $X$, if the ideal $J:=(I, F)$ truncates $H_{X}$.

(b) We say that $F$ is a strong truncator of $X$, if $F$ truncates $X$ and $J:=$ $(I, F)$ is a saturated ideal.

Remark 1.5. Since $X$ is a 0-dimensional scheme, an ideal of the form $J=$ $(I, F)$ is saturated if and only if $J$ is a perfect ideal, i.e. if and only if $A / J$ is a 1-dimensional Cohen-Macaulay ring. This last is the case if and only if $A / J$ contains a nonunit which is not a zero divisor.

We now give a simple criterion for a form to be a truncator. 
Proposition 1.6. Let $F \in A$ be homogeneous of degree $a_{X}+1$. The following statements are equivalent.

(a) $F$ is a truncator of $X$. $\mathbb{P}^{d}$.

(b) $(I: F)$ is an associated prime of $I$ which describes a $k$-rational point of

(c) $J:=(I, F)$ defines a subscheme of $X$ of degree $s-1$.

Moreover, there is a 1-1 correspondence (up to multiplication by nonzero scalars of $k$ and addition of elements of $\left.I_{a_{X}+1}\right)$ between truncators of $X$ and subschemes of $X$ of degree $s-1$.

Proof. First we show that (a) implies (b). Since $\operatorname{deg} F=a_{X}+1$, we find that $(I, F) / I \cong(A /(I: F))\left(-a_{X}-1\right)$. Also, since $F$ is a truncator of $X$, we have $\operatorname{dim}_{k}(J / I)_{n}=0$ for $n \leq a_{X}$ and $\operatorname{dim}_{k}(J / I)_{n}=1$ for $n>a_{X}$. Thus $\operatorname{dim}_{k}(A /(I: F))_{n}=0$ for $n \leq-1$ and $\operatorname{dim}_{k}(A /(I: F))_{n}=1$ for $n>-1$. In particular, $\operatorname{dim}_{k}(I: F)_{1}=d$, and so $(I: F) \supseteq\left(L_{1}, \ldots, L_{d}\right)$, where $L_{1}, \ldots, L_{d}$ are linearly independent linear forms. Comparison of the Hilbert functions of $A /(I: F)$ and $A /\left(L_{1}, \ldots, L_{d}\right)$ now yields the equality $(I: F)=\left(L_{1}, \ldots, L_{d}\right)$. Since the ideal $\left(L_{1}, \ldots, L_{d}\right)$ contains $I$ and has the same height $d$, it is an associated prime of $I$.

In order to prove that (b) implies (c), we use the assumption and calculate

$$
\operatorname{dim}_{k}(J / I)_{n}=\operatorname{dim}_{k}(A /(I: F))_{n-a_{X}-1}= \begin{cases}0 & \text { for } n \leq a_{X}, \\ 1 & \text { for } n>a_{X} .\end{cases}
$$

Thus we have $\operatorname{dim}_{k}(A / J)_{n}=s-1$ for $n>a_{X}$, and so $J$ defines a subscheme of $X$ of degree $s-1$, as we wanted.

Finally we show that (c) implies (a). Consider the exact sequences

$$
0 \rightarrow(J / I)_{n} \rightarrow(A / I)_{n} \rightarrow(A / J)_{n} \rightarrow 0
$$

for every $n \in \mathbb{Z}$. Since $x_{0}$ is not a zero divisor on $R=A / I$ and $J / I$ is an ideal of $R$, it follows that $\operatorname{dim}_{k}(J / I)_{n} \leq \operatorname{dim}_{k}(J / I)_{n+1}$ for all $n \in \mathbb{Z}$. By the definition of $a_{X}$, we have $\operatorname{dim}_{k}(A / I)_{n}=s$ for $n>a_{X}$. Since $J$ defines a subscheme of $X$ of degree $s-1$, we have $\operatorname{dim}_{k}(A / J)_{n}=s-1$ for all $n \gg 0$. Thus $\operatorname{dim}_{k}(J / I)_{n}=1$ for all $n \gg 0$. Now $F \notin I_{a_{X}+1}$, so $\operatorname{dim}_{k}(J / I)_{a_{X}+1}=1$. It follows that $\operatorname{dim}_{k}(J / I)_{n}=1$ for all $n>a_{X}$. Since $\operatorname{deg} F=a_{X}+1$, Proposition 1.3 gives that $F$ is a truncator of $X$.

For the last claim it suffices now to show that every subscheme of degree $s-1$ of $X$ corresponds to a truncator. So, let $Y$ be a subscheme of $X$ of degree $s-1$ and let $J$ be the ideal of $Y$ in $A$. Then $H_{X}\left(a_{X}+n\right)=s>H_{Y}\left(a_{X}+n\right)=s-1$ for all $n>0$. It follows that $\operatorname{dim}_{k}(J / I)_{a_{X}+n}=1$ for all $n \geq 1$. Hence a representative in $J$ of a generator for the vector space $(J / I)_{a_{X}+1}$ is a truncator of $X$.

Corollary 1.7. Let $X=\left\{P_{1}, \ldots, P_{s}\right\} \subseteq \mathbb{P}^{d}$ be a set of $s$ distinct $k$-rational points where $P_{i}$ corresponds to $\mathfrak{p}_{i} \subseteq A$. Suppose that $F \in A$ is homogeneous of degree $a_{X}+1$.

Then $F$ is a truncator of $X$ if and only if there is an $i \in\{1, \ldots, s\}$ such that $F \notin \mathfrak{p}_{i}$ and $F \in \mathfrak{p}_{1} \cap \cdots \cap \mathfrak{p}_{i-1} \cap \mathfrak{p}_{i+1} \cap \cdots \cap \mathfrak{p}_{s}$.

Example 1.8. Let $X=\left\{P_{1}, P_{2}, P_{3}, P_{4}\right\} \subseteq \mathbb{P}^{2}$ be the points $P_{1}=(1: 0: 1)$, $P_{2}=(1: 0: 0), P_{3}=(1: 1: 0)$ and $P_{4}=(1: 2: 0)$. 
Sketch, drawn in the affine space $D_{+}\left(X_{0}\right)$ :

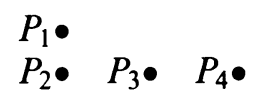

The Hilbert function of $X$ is $1344 \ldots$, and so $a_{X}=1$. Here $X_{0} X_{2} \in A_{2}$ is a truncator of $X$, but not a strong truncator.

Remark 1.9. It is worth recalling here that if $X$ is any 0 -dimensional subscheme of $\mathbb{P}^{d}$ which has degree $s$, then $X$ always contains subschemes of degree $s-1$. This is completely obvious when $X$ consists of $s$ distinct $k$-rational points. In the general case, it suffices to note that if $\mathfrak{p}$ is a prime ideal in $A$ and $\mathfrak{q}$ is $\mathfrak{p}$-primary, then if length $A_{\mathfrak{p}}\left(A_{\mathfrak{p}} / \mathfrak{q} A_{\mathfrak{p}}\right)=: r>1$ there is always a $\mathfrak{p}$-primary ideal $\mathfrak{q}^{\prime} \supset \mathfrak{q}$ having length $A_{\mathfrak{p}}\left(A_{\mathfrak{p}} / \mathfrak{q}^{\prime} A_{\mathfrak{p}}\right)=r-1$.

We now consider how to distinguish between truncators and strong truncators. Let $F \in A_{a_{X}+1}$ be a truncator of $X$, and let $f$ be the image of $F$ in $R$. As we have said, $F$ is a strong truncator if and only if $R /(f)$ is a CohenMacaulay ring. Since $R$ is a Cohen-Macaulay ring, this is equivalent to saying that $(f)$ does not have the irrelevant maximal ideal $\mathfrak{m}$ of $R$ as an associated prime. Put another way, $F$ is not a strong truncator if and only if there is an element $g \in R$ with $\mathfrak{m}=(f):(g)$. In this case we have $l g \in(f) \backslash\{0\}$ for any $l \in R_{1}$ which is not a zero divisor in $R$, and it follows that $\operatorname{deg} g \geq a_{X}$. On the other hand, since $\operatorname{dim}_{k}(f)_{n}=1$ for all $n \geq a_{X}+1$ and $l: R_{n} \rightarrow R_{n+1}$ is an injective map carrying $(f)_{n}$ onto $(f)_{n+1}$ for $n \geq a_{X}+1$, we are forced to conclude that $g \in R_{a_{X}}$ and $l g=c f$ for some $c \in k \backslash\{0\}$.

We summarize this discussion with the statement of the following proposition.

Proposition 1.10. Let $X$ be a 0 -dimensional subscheme of $\mathbb{P}^{d}$ and let $F \in A_{a_{X}+1}$ be a truncator of $X$. Then the following conditions are equivalent.

(a) $F$ is a strong truncator of $X$.

(b) $F \notin L A_{a_{X}}+I_{a_{X}+1}$ for every $L \in A_{1}$ whose image in $R$ is not a zero divisor.

(c) $F \notin \bigcap_{L}\left(L A_{a_{X}}+I_{a_{X}+1}\right)$, where the intersection ranges over those linear forms $L$ whose image in $R$ is not a zero divisor.

In [D] E. D. Davis asked whether any 0-dimensional subscheme of $\mathbb{P}^{d}$ has a strong truncator. We now show that this question has a negative answer.

Example 1.11. The ideal $I:=\left(X_{1}^{2}, X_{1} X_{2}, X_{2}^{3}-X_{0}^{2} X_{1}\right)$ defines a 0-dimensional subscheme $X$ of $\mathbb{P}^{2}$ having degree four which has a unique subscheme $Y$ of degree three but no strong truncator.

Proof. First observe that $\operatorname{rad}(I)=\left(X_{1}, X_{2}\right)$ and therefore $I$ has depth 2. Also, $I$ is generated by the $2 \times 2$-minors of the matrix $\left(\begin{array}{ccc}X_{2} & X_{1} & 0 \\ X_{0}^{2} & X_{2}^{2} & X_{1}\end{array}\right)$. So, $I$ is saturated (cf. [BV], (2.7)) and hence $\left(X_{1}, X_{2}\right)$-primary. Thus $I$ is the homogeneous ideal of a 0-dimensional subscheme $X$ of $\mathbb{P}^{2}$ which is concentrated at the point $(1: 0: 0)$.

It is easy to check that a $k$-basis of $R /\left(x_{0}\right)$ is given by $\left\{1, x_{1}, x_{2}, x_{2}^{2}\right\}$. Thus $H_{X}$ is $1344 \ldots$, and we see that $X$ has degree 4 and $a_{X}=1$. By Proposition 1.6, a form $F \in A_{2}$ is a truncator of $X$ if and only if $(I: F)=$ $\left(X_{1}, X_{2}\right)$. A simple calculation shows that this is equivalent to $F$ being of the 
form $F=c X_{0} X_{1}+G$, where $c \in k \backslash\{0\}$ and $G \in I_{2}$. Using Proposition 1.6, this means that $X$ has a unique subscheme of degree 3 , namely the one defined by $(I, F)=\left(X_{0} X_{1}, X_{1}^{2}, X_{1} X_{2}, X_{2}^{3}\right)$. Since this ideal is not saturated, $X$ does not have a strong truncator.

Remark 1.12. A description of those 0-dimensional subschemes of $\mathbb{P}^{d}$ which have strong truncators has, so far, eluded us. We have an example of two schemes, each of which does not have a strong truncator, but whose union has a strong truncator.

In spite of the negative nature of the example above, there is an important class of 0 -dimensional subschemes of $\mathbb{P}^{d}$ which do have strong truncators, namely sets of $k$-rational points. To see this, we first study how we can use truncators to describe the ring structure of $R$ from degree $a_{X}+1$ onward, if $X$ is a set of $k$-rational points.

Proposition 1.13. Let $X=\left\{P_{1}, \ldots, P_{s}\right\} \subseteq \mathbb{P}^{d}$ be a set of $s$ distinct $k$-rational points. By 1.6, for each $i \in\{1, \ldots, s\}$ we have a truncator $f_{i} \in R_{a_{X}+1}$ of $X$ corresponding to the subscheme $X \backslash\left\{P_{i}\right\}$. Since $X \subseteq D_{+}\left(X_{0}\right)$, we can write $P_{i}=$ $\left(1: p_{i 1}: \cdots: p_{i d}\right)$ with $p_{i j} \in k$ for $i=1, \ldots, s$. For $g \in R$ and $i \in\{1, \ldots, s\}$ we write $g\left(P_{i}\right):=G\left(1, p_{i 1}, \ldots, p_{i d}\right)$, where $G$ is any representative of $g$ in $A$.

(a) The elements $f_{1}, \ldots, f_{s}$ form a $k$-basis of $R_{a_{X}+1}$.

(b) By multiplying $f_{i}$ with a suitable element of $k$, we can assume that $f_{i}\left(P_{i}\right)=1$. For every $n \geq a_{X}+1$ and every element $g \in R_{n}$ we have a unique representation

$$
g=x_{0}^{n-a_{X}-1} g\left(P_{1}\right) f_{1}+\cdots+x_{0}^{n-a_{X}-1} g\left(P_{s}\right) f_{s} .
$$

Proof. First we show claim (a). We know that $H_{X}\left(a_{X}+1\right)=\operatorname{dim}_{k} R_{a_{X}+1}=s$. By 1.7 , we have $f_{i}\left(1, p_{j 1}, \ldots, p_{j d}\right)=0$ for $j \neq i$ and $f_{i}\left(1, p_{i 1}, \ldots, p_{i d}\right) \neq 0$. Suppose now that $g=c_{1} f_{1}+\cdots+c_{s} f_{s}=0$ for some $c_{i} \in k$. By evaluating $g$ at $P_{i}$ we find that $c_{i}=0$. That is enough to show that $f_{1}, \ldots, f_{s}$ are linearly independent, and so form a $k$-basis of $R_{a_{X}+1}$.

Now we prove part (b). From $\operatorname{dim}_{k} R_{n}=s$ for $n \geq a_{X}+1$ and from the injectivity of the multiplication by $x_{0}$ it follows that $R_{n}=x_{0}^{n-a_{X}-1} R_{a_{X}+1}$. Thus we have to show that every $g \in R_{a_{X}+1}$ satisfies $g=g\left(P_{1}\right) f_{1}+\cdots+g\left(P_{s}\right) f_{s}$. This follows from the observation that $g-g\left(P_{1}\right) f_{1}-\cdots-g\left(P_{s}\right) f_{s}$ vanishes at every point of $X$.

Using this proposition, we can now show that sets of $k$-rational points always have strong truncators.

Proposition 1.14. Let $X=\left\{P_{1}, \ldots, P_{s}\right\} \subseteq \mathbb{P}^{d}$ be a set of $s$ distinct $k$-rational points.

(a) $X$ has at least $s-H_{X}\left(a_{X}\right)+1$ linearly independent strong truncators. In particular, there is always a subscheme $Y$ of $X$ with $\operatorname{deg} Y=s-1$ and $H_{Y}=\operatorname{Trunc}\left(H_{X}\right)$.

(b) For any $r \in\{1, \ldots, s\}$ there is a subscheme $Y$ of $X$ consisting of $r$ points such that the Hilbert function of $Y$ is given by $H_{Y}(n)=\min \left\{r, H_{X}(n)\right\}$ for all $n \in \mathbb{Z}$.

Proof. First we show (a). With no loss in generality we can assume that $x_{i}:=$ $X_{i}+I$ is not a zero divisor in $R$ for each $i=0, \ldots, d$. Then the spaces $x_{i} R_{a_{X}}$ 
cannot all be equal, since they span $R_{a_{X}+1}$. Thus $\operatorname{dim}_{k}\left(x_{0} R_{a_{X}} \cap \cdots \cap x_{d} R_{a_{X}}\right)<$ $\operatorname{dim}_{k} R_{a_{X}}=H_{X}\left(a_{X}\right)$. In view of Propositions 1.10 and 1.13 , we conclude that $X$ has at least $s-H_{X}\left(a_{X}\right)+1$ strong truncators. Each of those corresponds to a subscheme $Y$ of $X$ with Hilbert function $\operatorname{Trunc}\left(H_{X}\right)$.

Claim (b) follows by repeated application of (a).

Compare this with Lemma (2.3) in [GMR]. The number $s-H_{X}\left(a_{X}\right)+1$ does not, however, appear to be a very good estimate for the number of strong truncators of $X$. It would be interesting to have sharper bounds. A. Sodhi, in [So], has made some observations on this question.

Remark 1.15. We had occasion to use the computer algebra system CoCoA (see [GN]) to help us with the initial explorations of the ideas in this paper. We found those explorations quite useful and want to mention some of the things one can do in that regard.

When $X=\left\{P_{1}, \ldots, P_{s}\right\}$ consists of $s$ distinct $k$-rational points, then the truncator of $X$ corresponding to $Y_{i}=X \backslash\left\{P_{i}\right\}$ can be found by looking at a generator of the 1-dimensional vector space $\left(I\left(Y_{i}\right) / I\right)_{a_{X}+1}$. In the next section we shall see that having a truncator for each of those subschemes $Y_{i}$ of $X$ is very useful for performing other computations with the scheme $X$.

\section{SeParators}

From now on and for the rest of this paper $X$ will always denote a 0 dimensional subscheme of $\mathbb{P}^{d}$ of degree $s$ which consists of $s$ distinct $k$ rational points $P_{1}, \ldots, P_{s}$. We shall continue to use the notations and assumptions given at the beginning of the first section. In particular, we assume $X \subseteq$ $D_{+}\left(X_{0}\right)$, so we can write $P_{i}=\left(1: p_{i 1}: \cdots: p_{i d}\right)$ with $p_{i j} \in k$ for $i=1, \ldots, s$. For $f \in R$ and $i \in\{1, \ldots, s\}$ we write $f\left(P_{i}\right):=F\left(1, p_{i 1}, \ldots, p_{i d}\right)$, where $F$ is any representative of $f$ in $A$.

Definition 2.1. Let $i \in\{1, \ldots, s\}$, let $n \geq 0$ and let $f \in R_{n}$. We say that $f$ is a separator of $P_{i}$ from $X \backslash\left\{P_{i}\right\}$, if $f\left(P_{i}\right) \neq 0$ and $f\left(P_{j}\right)=0$ for all $j \neq i$. The minimal number $n \geq 0$ such that there is a separator of $P_{i}$ from $X \backslash\left\{P_{i}\right\}$ is called the degree of $P_{i}$ in $X$ and denoted by $\operatorname{deg}_{X}\left(P_{i}\right)$.

We shall often just say that " $f$ is a separator of $P_{i}$ " when the enveloping scheme $X$ is clear from the context. We shall also refer to an element of $A_{n}$ as a separator of $P_{i}$ if it is a representative of a separator of $P_{i}$. Another way to phrase the definition of $\operatorname{deg}_{X}\left(P_{i}\right)$ is that it is the largest number $n$ such that any hypersurface of degree less than $n$ which contains $X \backslash\left\{P_{i}\right\}$ must also contain $P_{i}$.

Remark 2.2. A separator $f \in R_{n}$ of $P_{i}$ is unique up to multiplication by a scalar from $k \backslash\{0\}$.

Proof. Suppose $f, g \in R_{n}$ are two separators of $P_{i}$. If $n \geq a_{X}+1$, then $f$ and $g$ differ only by a scalar factor by $1.13(\mathrm{~b})$. If $n \leq a_{X}$ then multiply $f$ and $g$ by $x_{0}^{a_{X}+1-n}$ and get separators of $P_{i}$ again. Therefore, by $1.13(\mathrm{~b})$, the elements $x_{0}^{a_{x}+1-n} f$ and $x_{0}^{a_{x}+1-n} g$ only differ by a scalar factor. The fact that $x_{0}$ is not a zero divisor of $R$ finishes the proof. 
Proposition 2.3. Let $F \in A_{a_{X}+1}$. Then the following conditions are equivalent.

(a) $F$ is a separator of $P_{i}$ from $X \backslash\left\{P_{i}\right\}$.

(b) $F$ is a truncator of $X$ corresponding to $X \backslash\left\{P_{i}\right\}$.

Proof. This follows from Corollary 1.7.

Lemma 2.4. For every $i \in\{1, \ldots, s\}$ we have $\operatorname{deg}_{X}\left(P_{i}\right) \leq a_{X}+1$.

Proof. If we let $Y_{i}:=X \backslash\left\{P_{i}\right\}$, then $Y_{i}$ is a subscheme of $X$ of degree $s-1$, and so, as we observed in 1.6, there is a truncator of $X$ corresponding to $Y_{i}$. Using 2.3, it follows that $\operatorname{deg}_{X}\left(P_{i}\right) \leq a_{X}+1$ for every $i \in\{1, \ldots, s\}$.

Example 2.5. For $X=\left\{P_{1}, P_{2}, P_{3}, P_{4}\right\} \subseteq \mathbb{P}^{2}$ as in Example 1.8 we have $\operatorname{deg}_{X}\left(P_{1}\right)=1$ and $\operatorname{deg}_{X}\left(P_{i}\right)=2=a_{X}+1$ for $i=2,3,4$.

Proposition 2.6. Let $i \in\{1, \ldots, s\}$ and let $F \in A_{a_{X}+1}$ be a truncator corresponding to $X \backslash\left\{P_{i}\right\}$. The following statements are equivalent.

(a) $F$ is a strong truncator of $X$.

(b) $\operatorname{deg}_{X}\left(P_{i}\right)=a_{X}+1$.

(c) $Y_{i}:=X \backslash\left\{P_{i}\right\}$ has Hilbert function $H_{Y_{i}}=\operatorname{Trunc}\left(H_{X}\right)$.

Proof. Conditions (a) and (c) are equivalent by Definition 1.4. If $F$ is a strong truncator, then the homogeneous ideal of $Y_{i}$ is given by $(I, F)$. Therefore it agrees with $I$ up to degree $a_{X}$ and we must have $\operatorname{deg}_{X}\left(P_{i}\right) \geq a_{X}+1$. By Lemma 2.4, this means $\operatorname{deg}_{X}\left(P_{i}\right)=a_{X}+1$.

Conversely, suppose that $\operatorname{deg}_{X}\left(P_{i}\right)=a_{X}+1$ but $F$ is not a strong truncator of $X$. By Proposition 1.10, we can write $F=L G+H$ where $L \in A_{1}$ is a linear form whose image in $R$ is not a zero divisor, $G \in A_{a_{X}}$ and $H \in I_{a_{X}+1}$. Since $L(P) \neq 0$ for every $P \in X$, it follows that the image of $G$ in $R$ is a separator of $P_{i}$. This contradicts our assumption $\operatorname{deg}_{X}\left(P_{i}\right)=a_{X}+1$.

In view of Proposition 1.14 there are always at least $s-H_{X}\left(a_{X}\right)+1$ points of $X$ with degree $a_{X}+1$. Again this appears to be a rather bad bound. In practice, usually almost all points of $X$ have degree $a_{X}+1$. The extremal case is the most important situation in our investigations.

Definition 2.7. We say that $X$ is a Cayley-Bacharach scheme (CB-scheme), if one of the following equivalent conditions holds.

(a) Every $P \in X$ has degree $\operatorname{deg}_{X}(P)=a_{X}+1$.

(b) For any $P \in X$ we have $H_{X \backslash\{P\}}=\operatorname{Trunc}\left(H_{X}\right)$.

(c) The Hilbert function of $X \backslash\{P\}$ is independent of the choice of $P \in X$.

(d) Every hypersurface of degree less than $a_{X}+1$ which contains all but one point of $X$ must contain all the points of $X$.

Condition (d) is the one referred to by most authors discussing this property. For CB-schemes $X$, the notions of truncator, strong truncator and separator of degree $a_{X}+1$ all agree by 2.6 and the definitions.

Example 2.8. Every set of points in $\mathbb{P}^{d}$ which is a complete intersection of $d$ hypersurfaces is a CB-scheme. More generally, any arithmetically Gorenstein set of points in $\mathbb{P}^{d}$ is a CB-scheme (cf. [DGO]).

Example 2.9. If $C$ is a reduced and irreducible curve in $\mathbb{P}^{d+1}$, then $\mathrm{J}$. Harris $[\mathrm{H}]$ has shown that the points of a general hyperplane section of $C$ are in uniform position, i.e. any two subsets of $X$ with the same cardinality have the 
same Hilbert function. Harris' proof is for characteristic zero, but with some notable exceptions J. Rathmann [Ra] has also shown this result in characteristic $p \neq 0$. From the above definition we see that this notion of "uniform position" is equivalent to saying that the points of a general hyperplane section of $C$ form a CB-scheme and that this is true for every subset of those points.

Example 2.10. Let $X=\left\{P_{1}, \ldots, P_{8}\right\} \subseteq \mathbb{P}^{2}$ be given by $P_{1}=(1: 0: 2)$, $P_{2}=(1: 0: 1), P_{3}=(1: 0: 0), P_{4}=(1: 1: 2), P_{5}=(1: 1: 1)$, $P_{6}=(1: 1: 0), P_{7}=(1: 2: 1)$ and $P_{8}=(1: 2: 0)$.

Sketch in $D_{+}\left(X_{0}\right)$ :

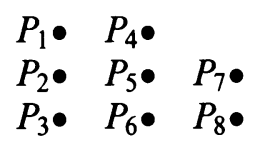

It is easy to check that $X$ is a CB-scheme but not a complete intersection. Also, $X$ is clearly not in uniform position.

Notice that Example 2.10 is a complete intersection less a point. Since we have already mentioned that complete intersections are CB-schemes, one is naturally led to ask which subschemes of a CB-scheme are also CB-schemes. For points in uniform position the answer is "all of them", but e.g. the scheme of Example 1.8 is a complete intersection minus two points and not a CB-scheme. The remainder of this section is devoted to investigating this question a bit further.

Notation. For the rest of this section we shall use the following notation. For each $P_{i} \in X$, we choose a separator $f_{i} \in R_{a_{X}+1}$ and a representative $F_{i}$ of $f_{i}$ in $A_{a_{X}+1}$. By Proposition 1.13(a), the set $\left\{f_{1}, \ldots, f_{s}\right\}$ is a $k$-basis of $R_{a_{X}+1}$.

Lemma 2.11. Let $r \in\{1, \ldots, s\}$, and let $J:=\left(I, F_{1}, \ldots, F_{r}\right)$. Then

$$
\operatorname{dim}_{k}(A / J)_{n}= \begin{cases}\operatorname{dim}_{k}(A / I)_{n} & \text { for } n \leq a_{X}, \\ s-r & \text { for } n>a_{X} .\end{cases}
$$

Proof. If $Y:=X \backslash\left\{P_{1}, \ldots, P_{r}\right\}$, then the elements of $J$ all vanish on $Y$. For $i \in\{r+1, \ldots, s\}$ the polynomial $F_{i}$ is not in $J$ by Proposition 1.13. So the ideal $J$ defines the subscheme $Y$ of $\mathbb{P}^{d}$ scheme-theoretically and $\operatorname{dim}_{k}(A / J)_{n}$ $=s-r$ for $n \gg 0$.

Now we claim that $k \cdot\left(l^{n} f_{1}\right) \oplus \cdots \oplus k \cdot\left(l^{n} f_{r}\right)=(J / I)_{a_{X}+1+n}$ for every $n \geq$ 0 and every $l \in R_{1}$ which is not a zero divisor. The case $n=0$ is true by hypothesis. The inclusion $\subseteq$ is then obviously true. If $n \geq 1$ and the inclusion is strict, then $\operatorname{dim}_{k}(J / I)_{a_{X}+1+m}>r$ for all $m \geq n$, because $l$ is not a zero divisor of $R$. This contradicts the fact that $\operatorname{dim}_{k}(J / I)_{a_{X}+1+m}=$ $\operatorname{dim}_{k}(A / I)_{a_{X}+1+m}-\operatorname{dim}_{k}(A / J)_{a_{X}+1+m}=r$ for $m \gg 0$. Thus the claim is true, and we get $\operatorname{dim}_{k}(A / J)_{a_{X}+1+n}=s-\operatorname{dim}_{k}(J / I)_{a_{X}+1+n}=s-r$ for $n \geq 0$ as desired.

Definition 2.12. If $J$ is a homogeneous ideal of $A$, we define the first difference function $\Delta H(A / J,-)$ of the Hilbert function of $A / J$ by

$$
\Delta H(A / J, n):=H(A / J, n)-H(A / J, n-1)
$$

for all $n \in \mathbb{Z}$. In the case $J=I$ we also write $\Delta H_{X}(n)$ or simply $h_{n}$ for $\Delta H(R, n)$. 
By Remark 1.1 we have $\Delta H_{X}(n) \neq 0$ if and only if $0 \leq n \leq a_{X}+1$. The last nonzero value of $\Delta H_{X}$ is denoted by $\Delta:=\Delta H_{X}\left(a_{X}+1\right)$. Notice that if $l \in R_{1}$ is not a zero divisor of $R$, then $\Delta H_{X}$ is the Hilbert function of $R / l R$.

Proposition 2.13. Let $r \in\{1, \ldots, \Delta\}$, let $Y:=X \backslash\left\{P_{1}, \ldots, P_{r}\right\}$ and choose $L \in A_{1}$ such that its image $l$ in $R$ is not a zero divisor. Then the following conditions are equivalent.

(a) The residue classes $\bar{f}_{1}, \ldots, \bar{f}_{r}$ are $k$-linearly independent in $(R / l R)_{a_{X}+1}$.

(b) $I(Y)=\left(I, F_{1}, \ldots, F_{r}\right)$.

(c) $H_{Y}(n)=H_{X}(n)$ for $n \leq a_{X}$ and $H_{Y}(n)=s-r$ for $n>a_{X}$.

Moreover, it is possible to renumber the points of $X$ such that the above conditions are satisfied.

Proof. First we show that (a) implies (b). As we observed in the proof of 2.11, the ideal $J:=\left(I, F_{1}, \ldots, F_{r}\right)$ defines $Y$ scheme-theoretically, so it suffices to prove that $J$ is saturated. We shall do this by showing that the image of $L$ in $A / J$ is not a zero divisor. Notice that $J_{n}=I_{n}$ for $n \leq a_{X}$, whence $L+J$ does not annihilate any homogeneous element of degree less than $a_{X}$ in $A / J$.

For $n>a_{X}$ we have $J_{n}=I_{n} \oplus k L^{n-a_{X}-1} F_{1} \oplus \cdots \oplus k L^{n-a_{X}-1} F_{r}$, as shown in the proof of Lemma 2.11. Suppose that $L G \in J_{n+1}$ for some $G \in A_{n}$. This means that $L\left(G-c_{1} L^{n-a_{X}-1} F_{1}-\cdots-c_{r} L^{n-a_{X}-1} F_{r}\right) \in I_{n+1}$ for some $c_{i} \in k$. But $l=L+I$ is not a zero divisor, so $G \in I_{n} \oplus k L^{n-a_{X}-1} F_{1} \oplus \cdots \oplus k L^{n-a_{X}-1} F_{r}=J_{n}$.

Finally we need that if $L G \in J_{a_{X}+1}$ for some $G \in A_{a_{X}}$, then $G \in I_{a_{X}}=J_{a_{X}}$. In this case write $L G=H+c_{1} F_{1}+\cdots+c_{r} F_{r}$ with $c_{i} \in k$ and $H \in I_{a_{X}+1}$. Now consider this equation in $R / l R$. We get $c_{1} \bar{f}_{1}+\cdots+c_{r} \bar{f}_{r}=0$. Since the elements $\bar{f}_{1}, \ldots, \bar{f}_{r}$ are linearly independent, we have $c_{1}=\cdots=c_{r}=0$. Thus $L G \in I_{a_{X}+1}$, which implies $G \in I_{a_{X}}$, as we wanted to show.

Now we prove that (b) implies (a). From 1.13 we know that $\left\{f_{1}, \ldots, f_{r}\right\}$ is a linearly independent set in $R_{a_{X}+1}$. So to prove the result it will suffice to show that $\left(k f_{1} \oplus \cdots \oplus k f_{r}\right) \cap l R_{a_{X}}=(0)$. Suppose that $l g=c_{1} f_{1}+\cdots+c_{r} f_{r}$ with $g \in R_{a_{X}}$ and $c_{i} \in k$ is in the intersection. From the hypothesis we get that $l g \in I(Y) / I$. Since the image of $l$ in $A / I(Y)$ is not a zero divisor, this implies that $g \in(I(Y) / I)_{a_{X}}$. But $I(Y)_{a_{X}}=I_{a_{X}}$, so $g=0$ and we are done.

Conditions (b) and (c) are equivalent by Lemma 2.11, because $J \subseteq I(Y)$ and both ideals agree if and only if their Hilbert functions agree.

The last claim follows from Proposition 1.14, or from the fact that $\left\{f_{1}, \ldots, f_{s}\right\}$ is a basis of $R_{a_{X}+1}$ which implies that $\left\{\bar{f}_{1}, \ldots, \bar{f}_{s}\right\}$ generates the $\Delta$-dimensional vector space $(R / l R)_{a_{X}+1}$.

As a first application of this proposition we can give a criterion for a subscheme $Y$ of $X$ to be a CB-scheme, if $Y$ is of the form $Y=X \backslash\left\{P_{1}, \ldots, P_{r}\right\}$ with $1 \leq r<\Delta$.

Corollary 2.14. Under the assumptions of the proposition let $\Delta \geq 2$ and $1 \leq r \leq$ $\Delta-1$. Then the following conditions are equivalent.

(a) $Y$ is a CB-scheme with Hilbert function $H_{Y}(n)=\min \left\{H_{X}(n), s-r\right\}$.

(b) For every $i \in\{r+1, \ldots, s\}$ the residue classes $\left\{\bar{f}_{1}, \ldots, \bar{f}_{r}, \bar{f}_{i}\right\}$ form a $k$-linearly independent set in $(R / l R)_{a_{X}+1}$.

Proof. Combine the proposition and 2.6. 
In the situation of Corollary 2.14 it is not always possible to choose a numbering of the points of $X$ such that $Y$ becomes a CB-scheme, even if $X$ is a CB-scheme to begin with. Our next example demonstrates this fact.

Example 2.15. Let $X:=\left\{P_{1}, \ldots, P_{6}\right\} \subseteq \mathbb{P}^{3}$, where $P_{1}=(1: 0: 0: 1), P_{2}=$ $(1: 0: 0:-1), P_{3}=(1: 0: 1: 0), P_{4}=(1: 0:-1: 0), P_{5}=(1: 1: 0: 0)$, and $P_{6}=(1:-1: 0: 0)$.

Sketch in $D_{+}\left(X_{0}\right)$ :

Here $H_{X}$ is $1466 \ldots$ and $\Delta H_{X}$ is $1320 \ldots$ Thus $a_{X}=1$ and $\Delta=2$. Also, $X$ is a CB-scheme, since any hyperplane contains at most four of the six points of $X$. But no subset of five points of $X$ forms a CB-scheme.

To see why this is so, observe that, since $X$ is a CB-scheme, all subsets $Y \subseteq X$ of degree five have $H_{Y}: 1455 \ldots$ and $a_{Y}=1$. Also, it is geometrically clear that each of those subsets has a point with $\operatorname{deg}_{Y}(P)=1$, namely the one opposite the point that we removed. Hence none of those $Y$ is a CB-scheme.

In the case $r=\Delta$ an application of Proposition 2.13 to our question yields a much more satisfactory answer.

Corollary 2.16. Suppose that $X$ is a CB-scheme. Renumber the points of $X$ such that $Y:=X \backslash\left\{P_{1}, \ldots, P_{\Delta}\right\}$ satisfies the conditions of 2.13 .

Then $Y$ is a CB-scheme.

Proof. From 2.13(c) we see that $a_{Y}=a_{X}-1$. Suppose that $Y$ is not a CBscheme, i.e. that there is a point $P_{i} \in Y$ and a form $G \in A_{a_{Y}}$ which is a separator of $P_{i}$ from $Y \backslash\left\{P_{i}\right\}$. Let $L \in A_{1}$ be a linear form which vanishes at $P_{i}$, but at no other point of $X$. Then $L G \in I(Y)_{a_{Y}+1}=\left(I, F_{1}, \ldots, F_{\Delta}\right)_{a_{X}}=I_{a_{X}}$ means that $G$ is a separator of $P_{i}$ from $X \backslash\left\{P_{i}\right\}$. That contradicts the fact that $X$ is a CB-scheme.

Corollary 2.17. If $X$ is a CB-scheme with $\Delta=1$, then $X \backslash\left\{P_{i}\right\}$ is a CB-scheme for every $i \in\{1, \ldots, s\}$.

Proof. Definition 2.7(b), Proposition 2.3 and Proposition 1.10 imply that every $\bar{f}_{i}$ is nonzero in $(R / l R)_{a_{X}+1}$. Hence the subscheme $X \backslash\left\{P_{i}\right\}$ satisfies the conditions of 2.13 . Now apply the previous corollary.

Example 2.18. If $X$ is a complete intersection of $d$ hypersurfaces of $\mathbb{P}^{d}$, or, more generally, if $X$ is arithmetically Gorenstein, then $\Delta=1$ and Corollary 2.17 applies. For instance, in this way one can see immediately that the scheme of Example 2.10 is a CB-scheme.

The following example shows that not every subset of $s-\Delta$ points of a CB-scheme is again a CB-scheme. Corollary 2.16 merely claims that there is at least one such subset. Of course, this raises the question whether one can characterize the CB-schemes having the property that all subschemes of degree $s-\Delta$ are CB-schemes again. We do not know an answer to this question. 
Example 2.19. Let $X:=\left\{P_{1}, \ldots, P_{8}\right\} \subseteq \mathbb{P}^{2}$ be given by $P_{1}=(1: 0: 3)$, $P_{2}=(1: 0: 2), P_{3}=(1: 0: 1), P_{4}=(1: 1: 1), P_{5}=(1: 2: 1)$, $P_{6}=(1: 0: 0), P_{7}=(1: 1: 0)$ and $P_{8}=(1: 2: 0)$.

Sketch in $D_{+}\left(X_{0}\right)$ :

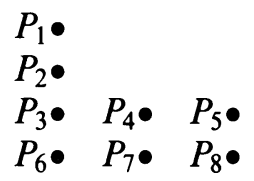

It is easy to see that $H_{X}$ is $13688 \ldots$, that $a_{X}=2$ and that $X$ is a CB-scheme with $\Delta=2$. Now $Y_{1}:=X \backslash\left\{P_{1}, P_{5}\right\}$ has Hilbert function $H_{Y_{1}}: 1366 \ldots$ and by 2.16 we conclude that $Y_{1}$ is a CB-scheme.

On the other hand, $Y_{2}:=X \backslash\left\{P_{5}, P_{8}\right\}$ and $a_{Y_{2}}=2$, and there is a conic which vanishes on $Y_{2} \backslash\left\{P_{4}\right\}$, but not on $P_{4}$. Thus $Y_{2}$ is not a CB-scheme. Notice also that $Y_{3}:=X \backslash\left\{P_{1}, P_{2}\right\}$ is a complete intersection and therefore a CB-scheme, but it does not have the same Hilbert function as $Y_{1}$.

Remark 2.20. Finally, we would like to make some comments about the computability of the notions of this section. On either of the computer algebra systems Macaulay or $\mathrm{CoCoA}$, it is possible to calculate the defining ideal of a given set of points $X$ and the Hilbert function $H_{X}$ of that set of points. For simplicity it is useful to assume that the points of $X$ do not lie on the hyperplane defined by $X_{0}$. If that is not the case at the outset one makes an appropriate linear change of coordinates.

To check if $X$ is a CB-scheme it suffices to check that for $n \leq a_{X}$ and any subset $Y$ of degree $s-1$ we have $(I(Y) / I)_{n}=0$. Another way to check this, which also gives information for other computations, is to find a truncator for each subscheme of $X$ of degree $s-1$. Then $X$ is a CB-scheme if and only if each truncator is a strong truncator. This can be tested by setting $X_{0}=0$ in the truncator and seeing if the result is in $I$ or not. Neither of these tests is very efficient. We shall see later (see $\S 4$ ) another approach to this problem.

To find out which subschemes of a CB-scheme are also CB-schemes, we use 2.14 and 2.16. We know that the residue classes of the truncators form a basis for the vector space $R_{a_{X}+1}$ (cf. 1.13). Using the truncators that have been found (cf. 1.15), set $X_{0}=0$ in each of them. We then need to discuss the linear independence of the residue classes of the resulting elements in $\left(R / x_{0} R\right)_{a_{X}+1}$. To apply 2.16 we just need to find $\Delta$ truncators which remain independent in $R / x_{0} R$. To apply 2.14 we need to find $r$ truncators which remain independent in $R / x_{0} R$ and have the property that every other truncator also remains independent of those $r$ truncators in $R / x_{0} R$. Both computer algebra systems mentioned earlier are very well suited to this kind of computation.

\section{The canonical module of a CB-scheme}

Throughout this section we continue to use the assumptions and conventions of the previous two sections. Since the projective coordinate ring $R$ of $X$ is a Cohen-Macaulay ring and $x_{0}$ is not a zero divisor of $R$, we have $k\left[x_{0}\right] \subseteq R$, and $R$ is a free $k\left[x_{0}\right]$-module of rank $s$. A homogeneous basis for $R$ can be found easily by lifting a homogeneous $k$-basis of $R / x_{0} R$. In this way we get 
an isomorphism of graded $k\left[x_{0}\right]$-modules

$$
R \cong \bigoplus_{i=0}^{a_{X}+1}\left(k\left[x_{0}\right](-i)\right)^{h_{i}}
$$

where $h_{i}:=\Delta H_{X}(i)$ as in 2.12. Now consider the graded $R$-module

$$
\operatorname{Hom}_{k\left[x_{0}\right]}\left(R, k\left[x_{0}\right]\right) \text {, }
$$

whose homogeneous component of degree $n$ consists of all graded $k\left[x_{0}\right]$-linear homomorphisms $\varphi: R \rightarrow k\left[x_{0}\right]$ with $\varphi\left(R_{m}\right) \subseteq k\left[x_{0}\right]_{m+n}$ for all $m \in \mathbb{Z}$. Its $R$-module structure is defined by setting $(r \varphi)\left(r^{\prime}\right):=\varphi\left(r r^{\prime}\right)$ for all $r^{\prime} \in R$.

We shall be particularly interested in the $R$-module

$$
\omega_{R}:=\operatorname{Hom}_{k\left[x_{0}\right]}\left(R, k\left[x_{0}\right]\right)(-1) .
$$

By [GW, 2.2.9], this is the canonical module of $R$. It is a finitely generated, graded $R$-module. First of all, let us compute its Hilbert function.

Proposition 3.1. For every $n \in \mathbb{Z}$ we have $H_{\omega_{R}}(-n)=s-H_{X}(n)$.

Proof. From the above description of $R$ we find

$$
\omega_{R} \cong \bigoplus_{i=0}^{a_{X}+1}\left(\operatorname{Hom}_{k\left[x_{0}\right]}\left(k\left[x_{0}\right](-i), k\left[x_{0}\right]\right)^{h_{i}}\right)(-1) \text {. }
$$

Therefore the $i$ th summand is canonically isomorphic to $k\left[x_{0}\right](i-1)^{h_{i}}$, and so $H_{\omega_{R}}(-n)=\sum_{i=0}^{a_{X}+1} h_{i} \operatorname{dim}_{k} k\left[x_{0}\right]_{i-1-n}$. On the other hand, since $H_{X}(n)=$ $\sum_{i=0}^{a_{X}+1} h_{i} \operatorname{dim}_{k} k\left[x_{0}\right]_{n-i}$, and since $i-1-n \geq 0$ if and only if $n-i<0$, we get

$$
H_{\omega_{R}}(-n)+H_{X}(n)=\sum_{i=0}^{a_{X}+1} h_{i}=s
$$

for every $n \in \mathbb{Z}$.

Our next goal is to give a characterization of CB-schemes in terms of the structure of their canonical module.

Lemma 3.2. Let $f_{i} \in R_{a_{X}+1}$ be a separator of $P_{i}$ for some $i \in\{1, \ldots, s\}$, let $g \in R_{n}$ for some $n \geq 0$, and let $\varphi \in\left(\omega_{R}\right)_{-a_{X}}$. Then

(a) $g f_{i}=g\left(P_{i}\right) x_{0}^{n} f_{i}$,

(b) $(g \varphi)\left(f_{i}\right)=g\left(P_{i}\right) x_{0}^{n} \varphi\left(f_{i}\right)$.

Proof. Claim (a) follows from 1.13(b) using the fact that the difference of the two expressions vanishes on $X$. Claim (b) follows immediately from (a), since $\varphi$ is $k\left[x_{0}\right]$-linear.

Remark 3.3. Let us consider Lemma 3.2(a) from another point of view. It gives a description of the multiplication in $R$ from degree $a_{X}+1$ onward in terms of the separators of degree $a_{X}+1$. Now recall that the integral closure $\widetilde{R}$ of $R$ in its total ring of fractions is of the form $\widetilde{R} \cong k\left[T_{1}\right] \times \cdots \times k\left[T_{s}\right]$ with polynomial variables $T_{1}, \ldots, T_{s}$. In one sense, we already know the multiplication of $R$ from degree $a_{X}+1$ onward, since $R$ and $\widetilde{R}$ agree in that range. Lemma 3.2(a) simply recognizes that structure and interprets it in $R$ itself. 
In particular, if $f_{1}, \ldots, f_{s} \in R_{a_{X}+1}$ are separators of $P_{1}, \ldots, P_{s}$ respectively, then we find that $f_{i} f_{j}=0$ for $j \neq i$ and $f_{i}^{2}=f_{i}(P) x_{0}^{a_{X}+1} f_{i}$ for $i=1, \ldots, s$.

Lemma 3.4. (a) There is a 1-1 correspondence between elements of $\left(\omega_{R}\right)_{-a_{X}}$ and $k$-linear maps from $R_{a_{X}+1}$ to $k$ which vanish on $x_{0} R_{a_{X}}$, given by $\left.\varphi \mapsto \varphi\right|_{R_{a_{X}+1}}$.

(b) Let $g \in R_{n}$ for some $n \geq 0$, and let $\varphi \in\left(\omega_{R}\right)_{-a_{X}}$. Then $g \varphi=0$ if and only if the restriction of $g \varphi$ to $R_{a_{X}+1}$ is the zero linear transformation.

(c) For $i=1, \ldots, s$ let $f_{i} \in R_{a_{X}+1}$ be a separator of $P_{i}$. An element $\varphi \in\left(\omega_{R}\right)_{-a_{X}}$ is faithful, i.e. $\operatorname{Ann}_{R}(\varphi)=(0)$, if and only if $\varphi\left(f_{i}\right) \neq 0$ for all $i \in\{1, \ldots, s\}$.

Proof. First we prove statement (a). Using the above description of $\omega_{R}$, an element $\varphi \in\left(\omega_{R}\right)_{-a_{X}}$ can be regarded as a graded $k\left[x_{0}\right]$-homomorphism $\varphi: R \rightarrow k\left[x_{0}\right]$ of degree $-a_{X}-1$. It follows that $\varphi\left(R_{n}\right)=0$ for $n=0, \ldots, a_{X}$ and that $\left.\varphi\right|_{R_{a_{X}+1}}: R_{a_{X}+1} \rightarrow k$ is a $k$-linear map which vanishes on $x_{0} R_{a_{X}}$.

Conversely, suppose that we are given a $k$-linear map $\psi: R_{a_{X}+1} \rightarrow k$ which vanishes on $x_{0} R_{a_{X}}$. We can extend $\psi$ to a $k$-linear map $\varphi: R \rightarrow k\left[x_{0}\right]$ in the following way. For $g \in R_{n}$ with $n \leq a_{X}$ define $\varphi(g):=0$. For $g \in R_{n}$ with $n>a_{X}+1$ write $g=x_{0}^{n-a_{X}-1} g^{\prime}$ using $1.13(\mathrm{~b})$ and define $\varphi(g):=$ $x_{0}^{n-a_{X}-1} \psi\left(g^{\prime}\right)$. Now the condition $\psi\left(x_{0} R_{a_{X}}\right)=0$ is precisely what we need to show that this definition makes $\varphi$ a $k\left[x_{0}\right]$-linear map which is homogeneous of degree $-a_{X}-1$. Hence $\varphi$ represents an element of $\left(\omega_{R}\right)_{-a_{X}}$.

For the proof of (b) suppose that $g \in R_{n}$ and $\varphi \in\left(\omega_{R}\right)_{-a_{X}}$ are such that the restriction of $g \varphi$ to $R_{a_{X}+1}$ is zero. If $h \in R_{m}$ with $m \leq a_{X}$, then $x_{0}^{a_{X}+1-m}(g \varphi)(h)=(g \varphi)\left(x_{0}^{a_{X}+1-m} h\right)=0$ implies that $(g \varphi)(h)=0$. If $h \in R_{m}$ with $m \geq a_{X}+1$, then we write $h=x_{0}^{m-a_{X}-1} h^{\prime}$ as in 1.13(b) and find $(g \varphi)(h)=x_{0}^{m-a_{X}-1}(g \varphi)\left(h^{\prime}\right)=0$, too. Hence $g \varphi=0$ as we wanted to show.

Finally we prove (c). If $\varphi$ is faithful and $\varphi\left(f_{i}\right)=0$ for some $i \in\{1, \ldots, s\}$, then $f_{i} \varphi$ restricted to $R_{a_{X}+1}$ is the zero homomorphism, because Lemma 3.2 yields $\left(f_{i} \varphi\right)\left(f_{j}\right)=f_{i}\left(P_{j}\right) x_{0}^{a_{X}+1} \varphi\left(f_{j}\right)=0$ for $j=1, \ldots, s$ and $\left\{f_{1}, \ldots, f_{s}\right\}$ is a $k$-basis of $R_{a_{X}+1}$. By (b), this implies that $f_{i} \varphi=0$ in contradiction to our assumption.

On the other hand, if $\varphi\left(f_{i}\right) \neq 0$ for $i=1, \ldots, s$ and $g \in R_{n} \backslash\{0\}$ for some $n \geq 0$, then there is at least one $i \in\{1, \ldots, s\}$ such that $g\left(P_{i}\right) \neq 0$. Using 3.2 again, this means $(g \varphi)\left(f_{i}\right)=g\left(P_{i}\right) x_{0}^{n} \varphi\left(f_{i}\right) \neq 0$. Therefore $g \varphi \neq 0$ and we are done.

Theorem 3.5. The following conditions are equivalent.

(a) $X$ is a CB-scheme.

(b) There is a faithful element in $\left(\omega_{R}\right)_{-a_{X}}$.

(c) A generic element of $\left(\omega_{R}\right)_{-a_{X}}$ is faithful.

(d) There is a homogeneous exact sequence of graded $R$-modules

$$
0 \rightarrow R \rightarrow \omega_{R}\left(-a_{X}\right) .
$$

Proof. Since (b) and (d) are clearly equivalent, and since (c) obviously implies (b), it suffices to prove that (a) implies (c) and (b) implies (a).

Suppose that $X$ is a CB-scheme, and take a generic element $\varphi \in\left(\omega_{R}\right)_{-a_{X}}$. By 1.10 and 2.6 we have $f_{i} \notin x_{0} R_{a_{X}}$ for $i=1, \ldots, s$. By 3.4(a) and since $\varphi$ 
is generic, it follows that $\varphi\left(f_{i}\right) \neq 0$ for $i=1, \ldots, s$. Thus $\varphi$ is faithful by 3.4(c).

If we start with a faithful element $\varphi \in\left(\omega_{R}\right)_{-a_{X}}$, we get $\varphi\left(f_{i}\right) \neq 0$ and $f_{i} \notin$ $x_{0} R_{a_{X}}$ from the lemma. By 1.10(c) and 2.6, it follows that $\operatorname{deg}_{X}\left(P_{i}\right)=a_{X}+1$ for $i=1, \ldots, s$, i.e. that $X$ is a CB-scheme.

Remark 3.6. There is also a nonreduced version of Theorem 3.5, which is proved in $[K 1, \S 2]$ using a similar technique. More precisely, there is a faithful element in $\left(\omega_{R}\right)_{-a_{X}}$ if and only if $X$ is locally Gorenstein and a CB-scheme.

One consequence of this theorem is that the property " $X$ is a CB-scheme" implies certain inequalities for its Hilbert function.

Corollary 3.7. Let $X$ be a $C B$-scheme.

(a) For all $i \in \mathbb{Z}$ we have $H_{X}(i)+H_{X}\left(a_{X}-i\right) \leq s$.

(b) For $i=0, \ldots, a_{X}+1$ we have $h_{0}+h_{1}+\cdots+h_{i} \leq h_{a_{X}+1-i}+\cdots+h_{a_{X}+1}$.

(c) For all $i, j$ with $0 \leq i \leq j \leq a_{X}+1$ we have $h_{0}+h_{1}+\cdots+h_{i} \leq$ $h_{j-i}+\cdots+h_{j}$.

Proof. From Proposition 3.5(d) and Proposition 3.1 we obtain

$$
H_{X}(i) \leq H_{\omega_{R}\left(-a_{X}\right)}(i)=H_{\omega_{R}}\left(i-a_{X}\right)=s-H_{X}\left(a_{X}-i\right) .
$$

This proves (a). Since $H_{X}(i)=h_{0}+h_{1}+\cdots+h_{i}$ and $s=h_{0}+h_{1}+\cdots+h_{a_{X}+1}$, claim (b) follows from (a). It remains to prove (c). By repeatedly applying Corollary 2.16 we find a subscheme $Y$ of $X$ of degree $H_{X}(j)$ with Hilbert function $H_{Y}(n)=\min \left\{H_{X}(n), H_{X}(j)\right\}$ for $n \in \mathbb{Z}$ and such that $Y$ is also a CB-scheme. Now (c) is nothing but (b) applied to $Y$.

Remark 3.8. Like 3.5, this corollary also has a generalization to 0-dimensional localiy Gorenstein schemes $X$. In fact, we do not even have to assume that $X$ is a CB-scheme, if we replace $a_{X}$ by the largest integer $n$ such that every hypersurface of degree $n$ containing a subscheme of degree $\operatorname{deg} X-1$ also contains $X$. That number is occasionally referred to as the Cayley-Bacharach number $c_{X}$ of $X$. In this case the inequalities that are valid are

$$
H_{X}(i)+H_{X}\left(c_{X}-i\right) \leq s \text { for } i=0, \ldots, a_{X}+1 .
$$

These results follow from [K2], 4.18 with $\mathscr{L}_{1}=\mathscr{O}_{\mathbb{P}^{d}}(i)$ and $\mathscr{L}_{2}=\mathscr{O}_{\mathbb{P}^{d}}\left(c_{X}-i\right)$.

Example 3.9. Let $X$ be a CB-scheme which does not lie on a hyperplane of $\mathbb{P}^{d}$. If $h_{a_{X}+1}=1$, then $h_{a_{X}} \geq d$. For if not, then $h_{a_{X}}+h_{a_{X}+1}<1+d=h_{0}+h_{1}$, and that contradicts Corollary 3.7(b).

Remark 3.10. If $X$ has the uniform position property, i.e. any two subsets of $X$ of the same cardinality have the same Hilbert function, then J. Harris and D. Eisenbud showed in [HE], 3.5 that

$$
H_{X}(i+j) \geq \min \left\{s, H_{X}(i)+H_{X}(j)-1\right\}
$$

for all $i, j \in \mathbb{Z}$. Note that if $i+j>a_{X}$, then $H_{X}(i+j)=s$ and the inequality holds trivially. So, the interesting case of (1) is $i+j \leq a_{X}$. In that case, $H_{X}(i+j)<s$ and (1) is equivalent to

$$
H_{X}(i+j) \geq H_{X}(i)+H_{X}(j)-1 .
$$


It is easy to see that (2) is the same as $3.7(\mathrm{c})$, if in those inequalities we replace $h_{j}$ by 1 . In particular, if we let $i+j=a_{X}$, then (2) is equivalent to

$$
h_{0}+h_{1}+\cdots+h_{i} \leq h_{a_{X}+1-i}+\cdots+h_{a_{X}}+1 .
$$

Again this is the same as 3.7(b) with $h_{a_{X}+1}$ replaced by 1 .

The stronger inequalities (2) are no longer true for arbitrary reduced CBschemes $X$, as the following example shows.

Example 3.11. Let $L_{1}$ and $L_{2}$ be two skew lines in $\mathbb{P}^{3}$, and let $P_{1}, \ldots, P_{4} \in$ $L_{1}$ and $P_{5}, \ldots, P_{8} \in L_{2}$ be $k$-rational points. Then it is easy to check that $X:=\left\{P_{1}, \ldots, P_{8}\right\}$ is a CB-scheme with Hilbert function $H_{X}: 14688 \ldots$ which does not satisfy (3).

This raises the question whether it is possible to find weaker conditions than the uniform position property which still imply the inequalities (2). One possible case is given by the following proposition.

Proposition 3.12. Suppose that $X$ is a CB-scheme with the property that for every $i \in\left\{0, \ldots, a_{X}\right\}$ there is a CB-scheme $Y_{i} \subseteq X$ of degree $\operatorname{deg} Y_{i}=H_{X}(i)+1$ and with Hilbert function $H_{Y_{i}}(n)=\min \left\{H_{X}(n), \operatorname{deg} Y_{i}\right\}$.

Then we have the inequalities $H_{X}(i+j) \geq H_{X}(i)+H_{X}(j)-1$ for $i, j \geq 0$ such that $i+j \leq a_{X}$.

Proof. Applying 3.7(a) to $Y_{i+j}$, we get the inequality $H_{X}(i+j)=\operatorname{deg} Y_{i+j}-1 \geq$ $H_{Y_{i+j}}(i)+H_{Y_{i+j}}\left(a_{Y_{i+j}}-i\right)-1=H_{X}(i)+H_{X}(j)-1$.

Another possibility is to ask for conditions that imply inequality (3) only. For instance, we have no example of a CB-scheme $X$ with $h_{a_{X}+1} \geq 2$ and the property that every subscheme $Y$ of degree $s-2$ has Hilbert function $H_{Y}(i)=\min \left\{s-2, H_{X}(i)\right\}$ which does not satisfy $(3)$.

In the last part of this section we want to point out the relation of the canonical module of $R$ to the conductor of $R$ in its integral closure. For $i=1, \ldots, s$ we set $d_{i}:=\operatorname{deg}_{X}\left(P_{i}\right)$ and we let $f_{i}$ be a separator of $P_{i}$ of degree $d_{i}$. Recall that the integral closure $\widetilde{R}$ of $R$ in its total ring of fractions is given by

$$
\widetilde{R} \cong R / \mathfrak{p}_{1} \times \cdots \times R / \mathfrak{p}_{s}=k\left[T_{1}\right] \times \cdots \times k\left[T_{s}\right],
$$

where $\mathfrak{p}_{i}$ is the ideal of $P_{i}$ in $R$ and $T_{i}$ is the image of $x_{0}$ in $R / \mathfrak{p}_{i}$.

Proposition 3.13. Let $\mathfrak{C}_{\widetilde{R} / R}$ denote the conductor of $R$ in $\widetilde{R}$.

(a) We have $\mathfrak{C}_{\widetilde{R} / R}=T_{1}^{d_{1}} k\left[T_{1}\right] \times \cdots \times T_{s}^{d_{s}} k\left[T_{s}\right]$ as an ideal of $\widetilde{R}$.

(b) We have $\mathfrak{C}_{\widetilde{R} / R}=\left(f_{1}, \ldots, f_{s}\right)$ as an ideal of $R$.

Proof. Part (a) is shown in [O]. We now use (a) to prove (b). The image of $f_{i}$ under the inclusion $R \subseteq \widetilde{R}$ is a scalar multiple of $\tilde{f}_{i}:=\left(0, \ldots, 0, T_{i}^{d_{i}}, 0, \ldots, 0\right)$. Since the $k\left[x_{0}\right]$-algebra structure of $\widetilde{R}$ is given by $x_{0} \mapsto\left(T_{1}, \ldots, T_{s}\right)$, those elements generate $\mathfrak{C}_{\widetilde{R} / R}$ as a $k\left[x_{0}\right]$-module, and the claim follows.

More precisely, note that from the above description of $\widetilde{R}$ we get $f_{i}=$ $f_{i}\left(P_{i}\right) \tilde{f}_{i}$ and $f_{i}^{2}=f_{i}\left(P_{i}\right) x_{0}^{d_{i}} f_{i}$. In view of 3.13 it is possible to make a simple connection between the conductor and the CB-property. 
Corollary 3.14. The scheme $X$ is a CB-scheme if and only if $\mathfrak{C}_{\widetilde{R} / R}=\bigoplus_{n \geq a_{X}+1} R_{n}$. Proof. Since $\operatorname{dim}_{k} \widetilde{R}_{n}=s$ if and only if $n \geq 0$, and since $\operatorname{dim}_{k} R_{n}=s$ if and only if $n \geq a_{X}+1$, we have $R_{n}=\widetilde{R}_{n}$ precisely for $n \geq a_{X}+1$. The ideal $\bigoplus_{n \geq a_{X}+1} R_{n}$ is an ideal of both $R$ and $\widetilde{R}$, and thus always contained in the conductor. By the proposition, we have equality if and only if $\operatorname{deg} f_{i}=a_{X}+1$ for $i=1, \ldots, s$, i.e. if and only if $X$ is a CB-scheme.

In other words, CB-schemes could have been defined as those schemes whose conductor is as small as possible. This characterization translates to 3.5 via Dedekind's formula for conductor and complementary module, which is the subject of the next proposition. Recall that the canonical module of $\widetilde{R}$ is given by $\omega_{\widetilde{R}} \cong \operatorname{Hom}_{k\left[x_{0}\right]}\left(\widetilde{R}, k\left[x_{0}\right]\right)(-1)$.

Proposition 3.15. For any set of $k$-rational points $X$ the following formula holds.

$$
\mathfrak{C}_{\widetilde{R} / R} \cdot \omega_{R}=\omega_{\widetilde{R}}
$$

Proof. For $r \in \mathfrak{C}_{\widetilde{R} / R}$ and $\varphi \in \omega_{R}$ we have $(r \varphi)(\widetilde{R})=\varphi(r \widetilde{R}) \subseteq \varphi(R) \subseteq k\left[x_{0}\right]$, so that $r \varphi \in \omega_{\widetilde{R}}$. Obviously this defines an inclusion $\mathfrak{C}_{\widetilde{R} / R} \cdot \omega_{R} \subseteq \omega_{\widetilde{R}}$.

To prove that this is an equality, we first compute the Hilbert function of $\omega_{\widetilde{R}}$. Since $\widetilde{R}$ is a free $k\left[x_{0}\right]$-module of rank $s$ with a homogeneous basis of degree 0 , it follows from the above description of $\omega_{\widetilde{R}}$ that $H_{\omega_{\widetilde{R}}}(n)=0$ for $n \leq 0$ and $H_{\omega_{\widetilde{R}}}(n)=s$ for $n \geq 1$. Therefore the projections

$$
\varphi_{i}: \widetilde{R} \cong k\left[T_{1}\right] \times \cdots \times k\left[T_{s}\right] \rightarrow k\left[T_{i}\right] \cong k\left[x_{0}\right],
$$

where $i \in\{1, \ldots, s\}$, form a $k\left[x_{0}\right]$-basis of $\omega_{\widetilde{R}}$ and it suffices to show that they lie in $\mathfrak{C}_{\widetilde{R} / R} \cdot \omega_{R}$.

Consider the epimorphism $\varepsilon: \operatorname{Hom}_{k\left[x_{0}\right]}\left(R, k\left[x_{0}\right]\right) \rightarrow \operatorname{Hom}_{k}\left(R /\left(x_{0}\right), k\right)$ induced by reduction modulo $x_{0}$. Since $f_{i}$ is a separator of $P_{i}$ of minimal degree, we must have $f_{i} \notin\left(x_{0}\right)$. Hence its image $\bar{f}_{i}$ in $\left(R /\left(x_{0}\right)\right)_{d_{i}}$ is not zero, and there is a homogeneous $k$-linear map $\bar{\psi}_{i}: R /\left(x_{0}\right) \rightarrow k$ of degree $-d_{i}$ such that $\bar{\psi}_{i}\left(\bar{f}_{i}\right) \neq 0$. Now lift $\bar{\psi}_{i}$ via $\varepsilon$ to a homogeneous $k\left[x_{0}\right]$-linear map $\psi_{i}: R \rightarrow k\left[x_{0}\right]$ of degree $-d_{i}$ with $\psi_{i}\left(f_{i}\right) \neq 0$. We conclude the proof by showing that there is a $\lambda_{i} \in k \backslash\{0\}$ such that $\varphi_{i}=\lambda_{i} f_{i} \psi_{i}$ as elements of $\omega_{\widetilde{R}}$. Then 3.13(b) yields $\varphi_{i} \in \mathfrak{C}_{\widetilde{R} / R} \cdot \omega_{R}$ as desired.

Write $f_{i}=f_{i}\left(P_{i}\right) \tilde{f}_{i}$ as in the note after 3.13. On one hand we have $\varphi_{i}\left(\tilde{f}_{j}\right)=$ 0 for $j \neq i$ and $\varphi_{i}\left(\tilde{f}_{i}\right)=x_{0}^{d_{i}}$. On the other hand we have $\left(f_{i} \psi_{i}\right)\left(\tilde{f}_{j}\right)=$ $f_{i}\left(P_{i}\right) \psi_{i}\left(f_{i} f_{j}\right)=0$ for $j \neq i$ and $\left(f_{i} \psi_{i}\right)\left(\tilde{f}_{i}\right)=f_{i}\left(P_{i}\right) \psi_{i}\left(f_{i}^{2}\right)=f_{i}\left(P_{i}\right)^{2} x_{0}^{d_{i}} \psi_{i}\left(f_{i}\right)$. We conclude that $\varphi_{i}=\lambda_{i} f_{i} \psi_{i}$ for $\lambda_{i}=f_{i}\left(P_{i}\right)^{2} \psi_{i}\left(f_{i}\right) \in k \backslash\{0\}$ as we wanted to show.

Remark 3.16. This proposition is also a consequence of [ $\mathrm{KW}, 4.32$ and $4.35(\mathrm{~b})]$. It is an easy exercise to use 3.15 to show that $3.5(\mathrm{c})$ and the condition on the conductor in 3.14 are equivalent. For example, if 3.5(c) is satisfied, then we must have $\operatorname{deg} f_{i}=a_{X}+1$ in 3.13(b), because otherwise 3.15 yields a nonzero element of $\left(\omega_{\widetilde{R}}\right)_{0}$. The other direction is left to the reader. 


\section{CB-SCHEMES AND LIAISON}

Continuing with our standing notation, we let $X$ be a set of $s$ distinct $k$ rational points in $\mathbb{P}^{d}$, we let $I(X)$ be the homogeneous ideal of $X$ in $A:=$ $k\left[X_{0}, \ldots, X_{d}\right]$, and $R:=A / I$ its projective coordinate ring.

In addition, we suppose that we have another set of $k$-rational points $Y$, disjoint from $X$, such that $Z:=X \cup Y$ is a complete intersection in $\mathbb{P}^{d}$. In other words, $X$ and $Y$ are linked by the complete intersection $Z$. Since most results in this section make use of the particular choice of $Y$, we would like to point out that at least one set of this type always exists (cf. Remark 4.11). Let $I(Y)$ be the homogeneous ideal of $Y$ in $A$, and let $\left\{G_{1}, \ldots, G_{d}\right\}$ be a homogeneous regular sequence in $A$ which defines $Z$, i.e. $I(Z)=\left(G_{1}, \ldots, G_{d}\right)$. It is well known that $I(X)=I(Z): I(Y)$ and $I(Y)=I(Z): I(Z)$.

In this situation the projective coordinate ring of $Z$ is $S:=A /\left(G_{1}, \ldots, G_{d}\right)$. Let us denote the images of $I(X)$ resp. $I(Y)$ in $S$ by $I$ resp. $J$. Thus we have $I=\operatorname{Ann}_{S}(J)$ and $J=\operatorname{Ann}_{S}(I)$. We also assume that the coordinates $\left\{X_{0}, \ldots, X_{d}\right\}$ of $\mathbb{P}^{d}$ are chosen in such a way that the image of $X_{0}$ in $S$ is not a zero divisor. We shall use "-" to denote residue classes modulo $X_{0}$.

Finally, let $\alpha_{X / Z}$ denote the initial degree of $I$, i.e.

$$
\alpha_{X / Z}=\min \left\{n \in \mathbf{N}: I_{n} \neq 0\right\}=\min \left\{n \in \mathbb{N}: I(X)_{n} \supsetneq\left(G_{1}, \ldots, G_{d}\right)_{n}\right\} .
$$

It may be worth noting that $\alpha_{X / Z}$ is not necessarily equal to

$$
\alpha_{X}=\min \left\{n \in \mathbb{N}: I(X)_{n} \neq 0\right\},
$$

but of course $\alpha_{X / Z} \geq \alpha_{X}$. Analogously, let $\alpha_{Y / Z}=\min \left\{n \in \mathbb{N}: J_{n} \neq 0\right\}$ be the initial degree of $J$.

The following preparatory results trace back to the work of Gröbner [G]. More modern versions are also contained in [DGO].

Lemma 4.1. For every ideal $\mathfrak{a}$ of $\bar{S}$ and every $n \geq 0$ we have

$$
\left(\operatorname{Ann}_{\bar{S}}\left(\mathfrak{a}_{n}\right)\right)_{a_{Z}+1-n}=\left(\operatorname{Ann}_{\bar{S}}(\mathfrak{a})\right)_{a_{Z}+1-n} .
$$

Proof. We only have to show that the left side is contained in the right one. Take an element $x \in \bar{S}_{a_{Z}+1-n}$ which annihilates $\mathfrak{a}_{n}$. Then $x \mathfrak{a}_{m}=0$ for every $m>n$ simply because $\bar{S}_{a_{Z}+1-n+m}=0$. Suppose that $m<n$ and $x y \neq 0$ for some $y \in \mathfrak{a}_{m}$. Since $\bar{S}$ is a 0 -dimensional local Gorenstein ring, its socle is $\bar{S}_{a_{z+1}}$, and therefore there is an element $z \in \bar{S}_{n-m}$ such that $x y z \in \bar{S}_{a_{z}+1} \backslash\{0\}$. This contradicts the assumption $x \mathfrak{a}_{n}=0$, so also $x \mathfrak{a}_{m}=0$ and we are done.

Lemma 4.2. For every ideal $\mathfrak{a}$ of $\bar{S}$ and every $n \in\left\{0, \ldots, a_{Z}+1\right\}$ we have

$$
\operatorname{dim}_{k}\left(\bar{S}_{n}\right)=\operatorname{dim}_{k}\left(\mathfrak{a}_{n}\right)+\operatorname{dim}_{k}\left(\operatorname{Ann}_{\bar{S}}(\mathfrak{a})\right)_{a_{Z}+1-n} .
$$

Proof. Let us consider the nondegenerate pairing $\bar{S}_{n} \times \bar{S}_{a_{Z}+1-n} \rightarrow \bar{S}_{a_{Z}+1} \cong k$. By the previous lemma, $\left(\operatorname{Ann}_{\bar{S}}(\mathfrak{a})\right)_{a_{Z}+1-n}$ is the orthogonal space to $\mathfrak{a}_{n}$ with respect to this pairing. The conclusion follows.

Proposition 4.3. Let $X, Y$, and $Z$ be as above.

(a) We have $\bar{I}=\operatorname{Ann}_{\bar{S}}(\bar{J})$ and $\bar{J}=\operatorname{Ann}_{\bar{S}}(\bar{I})$.

(b) For $n=0, \ldots, a_{Z}+1$ we have $\operatorname{dim}_{k}\left(\bar{S}_{n}\right)=\operatorname{dim}_{k}\left(\bar{I}_{n}\right)+\operatorname{dim}_{k}\left(\bar{J}_{a_{Z}+1-n}\right)$. 
(c) For $n=0, \ldots, a_{Z}+1$ we have $\Delta H_{Z}(n)=\Delta H_{X}(n)+\Delta H_{Y}\left(a_{Z}+1-n\right)$.

(d) We have $a_{Z}=a_{X}+\alpha_{Y / Z}=a_{Y}+\alpha_{X / Z}$.

Proof. For (a) it suffices to prove that $\bar{J}=\operatorname{Ann}_{\bar{S}}(\bar{I})$. Since $J=\operatorname{Ann}_{S}(I)$, we have $\bar{J} \subseteq \operatorname{Ann}_{\bar{S}}(\bar{I})$. Now consider the following equations.

$$
\begin{aligned}
\operatorname{dim}_{k}(\bar{S} / \bar{J}) & =\operatorname{deg} Y=\operatorname{deg} Z-\operatorname{deg} X=\operatorname{dim}_{k}(\bar{S})-\operatorname{dim}_{k}(\bar{S} / \bar{I}) \\
& =\operatorname{dim}_{k}\left(\bar{S} / \operatorname{Ann}_{\bar{S}}(\bar{I})\right) .
\end{aligned}
$$

The last equality follows from Lemma 4.2. We conclude that $\bar{J}=\operatorname{Ann}_{\bar{S}}(\bar{I})$.

Statement (b) is a consequence of (a) and Lemma 4.2. Claim (c) follows from (b), because $\Delta H_{X}(n)=\operatorname{dim}_{k}(\bar{S} / \bar{I})_{n}$ and $\Delta H_{Y}(n)=\operatorname{dim}_{k}(\bar{S} / \bar{J})_{n}$. From (c) we get that the initial degree of $\bar{I}$ is the least number $n$ such that $\Delta H_{Y}\left(a_{Z}+1-n\right) \neq$ 0 , i.e. it equals $a_{Z}-a_{Y}$. But the initial degree of $\bar{I}$ is also the initial degree of $I$, and we obtain (d).

Corollary 4.4. We have $\overline{I(Z)}_{a_{Z}+1}: \overline{I(Y)}_{\alpha_{Y / Z}}=\overline{I(X)}_{a_{X}+1}$.

Proof. When we read the desired equation in $\bar{S}$, we see that what we have to prove is $\left(\operatorname{Ann}_{\bar{S}}\left(\bar{J}_{\alpha_{Y / Z}}\right)\right)_{a_{Z}+1-\alpha_{Y / Z}}=\bar{I}_{a_{X}+1}$. This follows from 4.1, 4.3(a) and 4.3(d).

Now we shall apply these results and techniques to investigate the relation between a CB-scheme $X$ and a linked scheme $Y$. More precisely, we shall derive another characterization of CB-schemes using the ideal of a linked scheme.

Lemma 4.5. Suppose that $X$ is a CB-scheme. Let $P$ be a point of $X$, and let $F_{P} \in A_{a_{X}+1}$ be a separator of $P$ from $X \backslash\{P\}$.

Then $F_{P} \notin I(Z)_{a_{Z}+1}: I(Y)_{\alpha_{Y / Z}}$.

Proof. On the contrary, assume that $F_{P} \cdot I(Y)_{\alpha_{Y / Z}} \subseteq I(Z)_{a_{Z}+1}$. Then Corollary 4.4 shows that $\bar{F}_{P} \in \overline{I(X)}_{a_{X}+1}$. Hence $F_{P}$ is of the form $F_{P}=G_{P}+X_{0} H_{P}$ with $G_{P} \in I(X)_{a_{X}+1}$ and $H_{P} \in A_{a_{X}}$. But then also $H_{P}$ is a separator of $P$ from $X \backslash\{P\}$, in contradiction to $\operatorname{deg}_{X}(P)=a_{X}+1$. Thus the assumption was wrong and the claim follows.

Theorem 4.6. The following conditions are equivalent.

(a) $X$ is a $C B$-scheme.

(b) A generic element of $I(Y)_{\alpha_{Y / Z}}$ does not vanish at any point of $X$.

Proof. First we show that (a) implies (b). Let $P$ be a point of $X$, and let $F_{P} \in A_{a_{X}+1}$ be a separator of $P$ from $X \backslash\{P\}$. Then Lemma 4.5 implies that $F_{P} G \notin I(Z)_{a_{Z}+1}$ for a generic element $G$ of $I(Y)_{\alpha_{Y / Z}}$. Since $G$ vanishes at all points of $Y$ and $F_{P}$ vanishes at all points of $X \backslash\{P\}$, we must have $G(P) \neq 0$. Therefore a generic element of $I(Y)_{\alpha_{Y / Z}}$ does not vanish at any point of $X$.

Conversely, let $G \in I(Y)_{\alpha_{Y / Z}}$ be an element which does not vanish at any point of $X$. Let $P$ be a point of $X$, and let $F_{P} \in A$ be a separator of $P$ from $X \backslash\{P\}$ of minimal degree. Then $F_{P} G$ is a separator of $P$ from $Z \backslash\{P\}$. Since $Z$ is a CB-scheme (cf. 2.8), this yields $\operatorname{deg}\left(F_{P} G\right) \geq a_{Z}+1$. Thus $\operatorname{deg}_{X}(P)=\operatorname{deg}\left(F_{P}\right) \geq a_{Z}+1-\alpha_{Y / Z}=a_{X}+1$ by $4.3(\mathrm{~d})$, and therefore $X$ is a CB-scheme. 
Remark 4.7. Using the results of $\S 2.2$ in [GW], we obtain the following sequence of isomorphisms of graded $R$-modules.

$$
\begin{aligned}
\omega_{R}\left(-a_{X}\right) & \cong \operatorname{Hom}_{S}\left(R, \omega_{S}\right)\left(-a_{X}\right) \cong \operatorname{Hom}_{S}\left(S / I, S\left(a_{Z}\right)\right)\left(-a_{X}\right) \\
& \cong \operatorname{Hom}_{S}(S / I, S)\left(a_{Z}-a_{X}\right) \cong \operatorname{Ann}_{S}(I)\left(\alpha_{Y / Z}\right) \cong J\left(\alpha_{Y / Z}\right) .
\end{aligned}
$$

Based on this isomorphism $\omega_{R}\left(-a_{X}\right) \cong J\left(\alpha_{Y / Z}\right)$ one can prove that conditions 3.5(c) and 4.6(b) are equivalent. We chose to give a direct proof of Theorem 4.6 instead, because in this way we do not have to assume any knowledge about $\omega_{R}$ except that in our situation it is given by $\operatorname{Hom}_{k\left[x_{0}\right]}\left(R, k\left[x_{0}\right]\right)(-1)$.

Corollary 4.8. Let $Z$ be a 0-dimensional complete intersection in $\mathbb{P}^{d}$ which consists of distinct $k$-rational points. Let $Y$ be a subset of $Z$ such that $I(Y)$ is generated by its elements of degree less than or equal to $\alpha_{Y / Z}$.

Then $X:=Z \backslash Y$ is a $C B$-scheme.

Proof. If $X$ is not a CB-scheme, then a generic element of $I(Y)_{\alpha_{Y / Z}}$ vanishes at some point of $X$. Hence there is a point of $X$ at which all forms of $I(Y)_{\alpha_{Y / Z}}$ vanish. This is impossible, since $I(Y)$ is generated in degree less than or equal to $\alpha_{Y / Z}$.

Theorem 4.6 and Corollary 4.8 provide us with handy ways to check geometrically whether a given set of points forms a CB-scheme.

Example 4.9. Let $Z$ be the complete intersection of the two cubics $G_{1}=X_{1}^{3}-$ $X_{0}^{2} X_{1}$ and $G_{2}=X_{2}^{3}-X_{0}^{2} X_{2}$ in $\mathbb{P}^{2}$.

Sketch:

$$
\begin{array}{lll}
P_{1} \bullet & P_{2} \bullet & P_{3} \\
P_{4} \bullet & P_{5} \bullet & P_{6} \\
P_{7} \bullet & P_{8} \bullet & P_{9}
\end{array}
$$

Then $Z \backslash\left\{P_{i}\right\}$ is a CB-scheme for every $i \in\{1, \ldots, 9\}$, because a generic line through $P_{i}$ does not contain any point of $Z \backslash\left\{P_{i}\right\}$. (Compare this with 2.17.)

The scheme $Z \backslash\left\{P_{1}, P_{2}\right\}$ is not a CB-scheme, because there is only one line containing $\left\{P_{1}, P_{2}\right\}$ and this line also contains $P_{3}$. On the other hand, the scheme $Z \backslash\left\{P_{2}, P_{4}\right\}$ is a CB-scheme, because the line through $P_{2}$ and $P_{4}$ does not contain any point of $Z \backslash\left\{P_{2}, P_{4}\right\}$.

Finally, the scheme $Z \backslash\left\{P_{2}, P_{3}, P_{5}, P_{6}\right\}$ is a CB-scheme, since the ideal of the scheme $Y:=\left\{P_{2}, P_{3}, P_{5}, P_{6}\right\}$ has initial degree two and is generated by forms of degree two, so that we can apply 4.8. In the same manner one can also show that the scheme of Example 2.19 is a CB-scheme.

The theorem can also be used to formulate characterizations of CB-schemes which are well suited for the application of computer algebra programs.

Corollary 4.10. The following conditions are equivalent.

(a) $X$ is a CB-scheme.

(b) $I(Z): I(Y)_{\alpha_{Y / Z}}=I(X)$.

(c) $\operatorname{Ann}_{S}\left(J_{\alpha_{Y / Z}}\right)=I$.

(d) $I(Z)_{a_{Z}}: I(Y)_{\alpha_{Y / Z}}=I(X)_{a_{X}}$.

(e) $\left(\operatorname{Ann}_{S}\left(J_{\alpha_{Y / Z}}\right)\right)_{a_{X}}=I_{a_{X}}$.

(f) $H\left(A /\left(I(Z):(I(Z): I(X))_{\alpha_{Y / Z}}\right), a_{Z}-\alpha_{Y / Z}\right)=H_{X}\left(a_{Z}-\alpha_{Y / Z}\right)$. 
Proof. First we show that (a) implies (b). We only have to prove that the left side is contained in the right one. Let $F$ be an element of $I(Z): I(Y)_{\alpha_{Y / Z}}$. Then $F G \in I(Z)$ for every $G \in I(Y)_{\alpha_{Y / Z}}$. Using Theorem 4.6 we deduce that $F$ must vanish at every point of $X$.

Conditions (b) and (c) are obviously equivalent. In view of formula 4.3(d) it is also clear that (b) implies (d). Condition (e) is a restatement of (d) in $S$ and therefore equivalent to (d). Conditions (d) and (f) are equivalent, because $I(Z)_{a_{Z}}:(I(Z): I(X))_{\alpha_{Y / Z}} \supseteq I(X)_{a_{X}}$ is always true, so the equality of those vector spaces follows from the equality of their dimensions.

Finally we have to show that (d) implies (a). Assume that $X$ is not a CBscheme, i.e. that there is a point $P$ in $X$ and a separator $F_{P}$ of $P$ from $X \backslash\{P\}$ of degree $a_{X}$. Then $F_{P} \notin I(Z)_{a_{Z}}: I(Y)_{\alpha_{Y / Z}}$ by hypothesis. Therefore, if we take a generic element $G$ of $I(Y)_{\alpha_{Y / Z}}$, then $F_{P} G$ is a separator of $P$ from $Z \backslash\{P\}$ of degree $a_{X}+\alpha_{Y / Z}=a_{Z}$. This contradicts the fact that $Z$ is a CB-scheme.

Remark 4.11. Suppose that we are given a 0-dimensional subscheme $X=$ $\left\{P_{1}, \ldots, P_{s}\right\}$ of $\mathbb{P}^{d}$ which consists of $k$-rational points. How can we decide whether $X$ is a CB-scheme? Two not very efficient ways have been discussed in 2.20. The following method is based on Corollary 4.10 and works much better.

We assume that we are either given $I(X)$ directly, or we have computed it from the coordinates of the points of $X$ by intersecting the corresponding prime ideals. We also need a homogeneous regular sequence $\left\{G_{1}, \ldots, G_{d}\right\}$ in $I(X)$ which defines a 0 -dimensional complete intersection $Z$ containing $X$. In principle, we can always write $P_{i}=\left(1: p_{i 1}: \cdots: p_{i d}\right)$ and use $G_{i}:=$ $\left(X_{i}-p_{1 i} X_{0}\right) \cdots\left(X_{i}-p_{s i} X_{0}\right)$ for $i=1, \ldots, d$. This choice has the disadvantage of producing polynomials of high degree, but it has the advantages of being computationally trivial, if the points are given via their coordinates, and of producing a Gröbner basis with respect to any term ordering that starts with the degree in $X_{1}, \ldots, X_{d}$ (cf. [Ro, 9.2(b)]). From here we can proceed in the following way.

First of all, no matter how we got the regular sequence, we have $I(Z)$ and $a_{Z}=\operatorname{deg} G_{1}+\cdots+\operatorname{deg} G_{d}-d$. Then we start the computation of $I(Z): I(X)$. This is done by computing a suitable Gröbner basis, and we recall that the computation proceeds degree after degree. As soon as an element is found, we check whether it is in $I(Z)$. This operation is computationally easy, if we have a Gröbner basis of $I(Z)$ (see above). As soon as we get an element which is not in $I(Z)$, its degree is $\alpha_{Y / Z}$. So we can stop the computation of $I(Z): I(X)$ at this degree and we have $(I(Z): I(X))_{\alpha_{Y / Z}}$. Now we compute $\left(I(Z):(I(Z): I(X))_{\alpha_{Y / Z}}\right)$ by means of a Gröbner basis which is truncated in degree $a_{Z}-\alpha_{Y / Z}$ and we check formula 4.10 (f) by simply computing the dimensions of the respective vector spaces.

To illustrate this method, we explicitly describe how we use it to prove that the scheme $X$ in Example 2.19 is indeed a CB-scheme. From the coordinates of the points we compute that

$$
\begin{aligned}
& I(X)=\left(X_{1} X_{2}^{2}-X_{0} X_{1} X_{2},\right. X_{1}^{3}-3 X_{0} X_{1}^{2}+2 X_{0}^{2} X_{1}, \\
&\left.X_{2}^{4}-6 X_{0} X_{2}^{3}+11 X_{0}^{2} X_{2}^{2}-6 X_{0}^{3} X_{2}\right) .
\end{aligned}
$$

Now we choose $G_{1}=X_{1}\left(X_{1}-X_{0}\right)\left(X_{1}-2 X_{0}\right)$ and 


$$
G_{2}=X_{2}\left(X_{2}-X_{0}\right)\left(X_{2}-2 X_{0}\right)\left(X_{2}-3 X_{0}\right)
$$

and we set $I(Z):=\left(G_{1}, G_{2}\right)$ and get $a_{Z}=4$. We start computing $(I(Z): I(X))$ and we get $\left(X_{2}^{2}-5 X_{0} X_{2}+6 X_{0}^{2}, X_{1}^{2}-3 X_{0} X_{1}+2 X_{0}^{2}\right)$ in degree two. Since $I(X)$ starts in degree three, we get $\alpha_{Y / Z}=2$ and $a_{Z}-\alpha_{Y / Z}=2$. We compute $\left(I(Z):(I(Z): I(X))_{2}\right)_{2}$ and we get $(0)$. Also $I(X)_{2}=(0)$, so that we can conclude that $X$ is a CB-scheme.

\section{APPLICATIONS TO HYPERPLANE SECTIONS OF CURVES}

As an application of our results, we would like to show how they fit with those in a recent paper of $R$. P. Stanley. Recall that we are always working over a field $k$ of arbitrary characteristic. For the proof of the next proposition we need to use the description of $\omega_{R}$ via local cohomology as outlined for instance in [GW, Chapter 2].

Proposition 5.1. Let $k$ be algebraically closed, and let $C$ be an integral, arithmetically Cohen-Macaulay curve in $\mathbb{P}_{k}^{d+1}$.

Then the general hyperplane section $X$ of $C$ is a CB-scheme.

Proof. Let $R$ be the projective coordinate ring of $C$ and let $\omega_{R}$ be its canonical module. Then $\omega_{R}$ is a 2-dimensional Cohen-Macaulay $R$-module. We define $a_{R}:=-\min \left\{n \in \mathbb{Z}:\left(\omega_{R}\right)_{n} \neq 0\right\}$, and we let $x$ be a nonzero element of $\left(\omega_{R}\right)_{-a_{R}}$. Since $\operatorname{Ass}(R x) \subseteq \operatorname{Ass}\left(\omega_{R}\right)=0$, we get $\operatorname{dim}\left(R / \operatorname{Ann}_{R}(x)\right)=$ $\max \{\operatorname{dim} R / \mathfrak{p}: \mathfrak{p} \in \operatorname{Ass}(R x)\}=2$. Therefore we have $\operatorname{Ann}_{R}(x)=0$. Now consider the homogeneous exact sequence of graded $R$-modules

$$
0 \rightarrow R\left(a_{R}\right) \stackrel{x}{\rightarrow} \omega_{R} \rightarrow \omega_{R} / R x \rightarrow 0
$$

and the resulting long exact sequence of local cohomology modules

$$
\begin{aligned}
\cdots & \rightarrow H_{\mathfrak{m}}^{0}\left(\omega_{R}\right) \rightarrow H_{\mathfrak{m}}^{0}\left(\omega_{R} / R x\right) \rightarrow H_{\mathfrak{m}}^{1}(R)\left(a_{R}\right) \stackrel{x}{\rightarrow} H_{\mathfrak{m}}^{1}\left(\omega_{R}\right) \\
& \rightarrow H_{\mathfrak{m}}^{1}\left(\omega_{R} / R x\right) \rightarrow H_{\mathfrak{m}}^{2}(R)\left(a_{R}\right) \stackrel{x}{\rightarrow} H_{\mathfrak{m}}^{2}\left(\omega_{R}\right) \rightarrow H_{\mathfrak{m}}^{2}\left(\omega_{R} / R x\right) \rightarrow 0
\end{aligned}
$$

where $\mathfrak{m}$ denotes the homogeneous maximal ideal of $R$.

Since $H_{\mathrm{m}}^{0}\left(\omega_{R}\right)=H_{\mathfrak{m}}^{1}(R)=0$, we get $\operatorname{depth}_{R}\left(\omega_{R} / R x\right) \geq 1$. Since the isomorphisms $H_{\mathrm{m}}^{2}(R) \cong \operatorname{Hom}_{k}\left(\omega_{R}, k\right)$ and $H_{\mathrm{m}}^{2}\left(\omega_{R}\right) \cong \operatorname{Hom}_{k}(R, k)$ identify the multiplication map $\mu_{x}: H_{\mathfrak{m}}^{2}(R)\left(a_{R}\right) \rightarrow H_{\mathfrak{m}}^{2}\left(\omega_{R}\right)$ with $\operatorname{Hom}_{k}\left(\mu_{x}, k\right)$ : $\operatorname{Hom}_{k}\left(\omega_{R}\left(-a_{R}\right), k\right) \rightarrow \operatorname{Hom}_{k}(R, k)$, we see that $\mu_{x}$ is surjective and thus $H_{\mathrm{m}}^{2}\left(\omega_{R} / R x\right)=0$. Hence $\operatorname{dim}\left(\omega_{R} / R x\right) \leq 1 \leq \operatorname{depth}_{R}\left(\omega_{R} / R x\right)$, and thus $\omega_{R} / R x$ is a 1 -dimensional Cohen-Macaulay $R$-module. In particular, this implies that a general linear form $l \in R_{1}$ is not a zero divisor on $\omega_{R} / R x$.

Now let $S:=R /(l)$, and let $X:=C \cap \mathscr{V}(l)$. Then $X$ is a general hyperplane section of $C$ and $S$ is its projective coordinate ring. Since $l$ is not a zero divisor of $R$, we get $\omega_{S} \cong\left(\omega_{R} / l \omega_{R}\right)(1)$ and $a_{X}=-\min \left\{n \in \mathbb{Z}:\left(\omega_{S}\right)_{n} \neq 0\right\}$ $=a_{R}+1$. Using these identities we construct the following commutative diagram. 


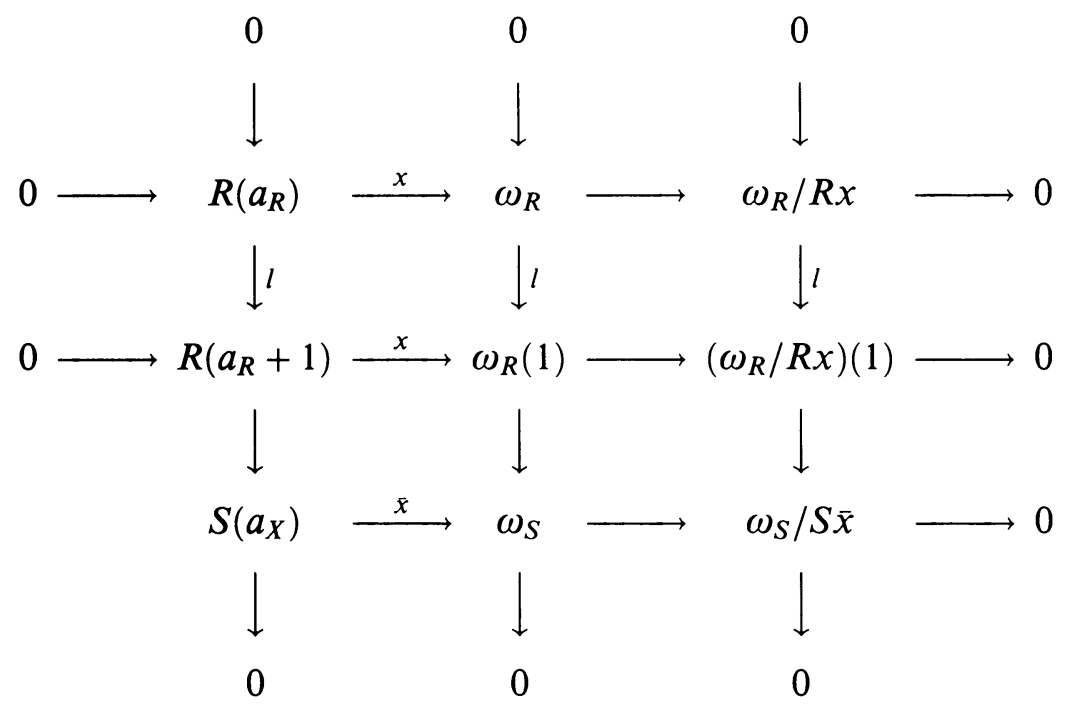

An application of the snake lemma to this diagram yields $\operatorname{Ann}_{S}(\bar{x})=0$, i.e. $\bar{x}$ is a faithful element in $\omega_{S}\left(-a_{X}\right)$. By Theorem 3.5, this implies that $X$ is a CB-scheme.

In particular, we can apply Corollary 3.7 and get the following inequalities for the Hilbert function of a general hyperplane section of a Cohen-Macaulay curve.

Corollary 5.2. In the situation of the proposition let $h_{i}:=\Delta H_{X}(i)$ for all $i \geq 0$.

Then $h_{0}+h_{1}+\cdots+h_{i} \leq h_{j-i}+\cdots+h_{j}$ for all $0 \leq i \leq j \leq a_{X}+1$.

Now let $k$ be an arbitrary infinite field again, and let $R$ be a standard graded $k$-algebra, i.e. $R_{0}=k$ and $R=k\left[R_{1}\right]$. When $R$ is a Cohen-Macaulay ring, we can define its $h$-vector $\left(h_{0}, \ldots, h_{t}\right)$ by $h_{i}:=\Delta^{\operatorname{dim} R} H(R, i)$ and $t:=$ $\max \left\{i \in \mathbb{N}: h_{i} \neq 0\right\}$. Notice that this definition agrees with 2.12, if $R$ is the projective coordinate ring of a set of $k$-rational points.

The next corollary gives more inequalities on the $h$-vector of a CohenMacaulay integral domain $R$ than [St, 2.1], under the additional assumption that $R$ is standard graded.

Corollary 5.3. If $\boldsymbol{R}$ is a standard graded Cohen-Macaulay integral domain over an infinite field $k$, then its $h$-vector satisfies

$$
h_{0}+h_{1}+\cdots+h_{i} \leq h_{j-i}+\cdots+h_{j}
$$

for all $0 \leq i \leq j \leq t$.

Proof. Since the $h$-vector does not change when we enlarge the ground field, we may assume that $k$ is algebraically closed. Since the case $\operatorname{dim} R=0$ is impossible, and since the case $\operatorname{dim} R=1$ is only possible for $R=k[X]$, we can also assume that $\operatorname{dim} R \geq 2$. If $\delta:=\operatorname{dim} R \geq 3$, then we find a regular sequence $x_{1}, \ldots, x_{\delta-2} \in R_{1}$ such that $R /\left(x_{1}, \ldots, x_{\delta-2}\right)$ is a Cohen-Macaulay domain of dimension two with the same $h$-vector (cf. [St, 3.3]). Thus we can assume that $\operatorname{dim} R=2$. In this case $R$ is the projective coordinate ring of an integral Cohen-Macaulay curve in $\mathbb{P}^{d+1}$, where $d+2$ is the minimal number 
of generators of the homogeneous maximal ideal of $R$. Since $R$ is CohenMacaulay, its $h$-vector agrees with the $h$-vector of a general hyperplane section $X$ of $C$. Now an application of Corollary 5.2 finishes the proof.

Let us also compare the inequalities of Corollary 5.2 with Harris' inequalities for the Hilbert function of hyperplane sections of curves. For this we let $k$ be an algebraically closed field again, and let $C \subseteq \mathbb{P}^{d+1}$ be an integral curve. In this case char $k=0$, Harris has shown in $[\mathrm{H}]$ that the points of a general hyperplane section of $C$ are in uniform position, and therefore the stronger inequalities 3.10.2 hold. But in characteristic $p>0$, Harris' result is true only with some additional hypothesis, as demonstrated by J. Rathmann in [Ra]. The following example, due to Rathmann, illustrates that although Proposition 5.1 yields weaker inequalities, it applies in some previously not covered cases.

Example 5.4. Let char $k=p>0$, let $q=p^{n}$ for some $n>0$, and let $C \subseteq \mathbb{P}^{d+1}$ be the curve defined by

$$
C:=\mathscr{V}\left(X_{0}^{q}-X_{1} X_{d+1}^{q-1}, X_{1}^{q}-X_{2} X_{d+1}^{q-1}, \ldots, X_{d-1}^{q}-X_{d} X_{d+1}^{q-1}\right) .
$$

Then $C$ is an integral complete intersection curve whose general hyperplane section is isomorphic to the set of $\mathbb{F}_{q}$-rational points of the $d$-dimensional affine space over $\mathbb{F}_{q}$ (see $[\mathrm{Ra}, 1.2]$ for details). By Proposition 5.1 we see that $X$ is a CB-scheme and therefore its $h$-vector satisfies the inequalities given in 5.2. But obviously $X$ is not a set of points in uniform position.

With the pervasiveness of the CB-property for general hyperplane sections of Cohen-Macaulay curves in arbitrary characteristic one could wonder, if every hyperplane section of a Cohen-Macaulay curve is a CB-scheme. The following example shows that this is not the case.

Example 5.5. Let $k$ be a field of characteristic zero. Consider the 0-dimensional reduced subscheme $X=\left\{P_{1}, \ldots, P_{7}\right\}$ of $\mathbb{P}^{2}$ with $P_{1}=\left(1: 0: 0, P_{2}=(1:\right.$ $0: 1), P_{3}=(1: 0: 2), P_{4}=(1: 1: 0), P_{5}=(1: 1: 1), P_{6}=(1: 1: 2)$ and $P_{7}=(1: 2: 0)$.

Sketch:

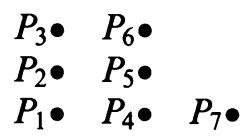

Here $X$ is not a CB-scheme (cf. 4.9), but we shall show that there is an integral, arithmetically Cohen-Macaulay curve $C$ such that $X$ is one of its hyperplane sections. It is not hard to see that the ideal of $X$ in $k\left[X_{0}, X_{1}, X_{2}\right]$ is given by

$$
I=\left(X_{1}\left(X_{1}-X_{0}\right)\left(X_{1}-2 X_{0}\right), X_{1}\left(X_{1}-X_{0}\right) X_{2}, X_{2}\left(X_{2}-X_{0}\right)\left(X_{2}-2 X_{0}\right)\right) \text {. }
$$

The resolution of the ideal sheaf $\mathscr{T}_{X}$ of $X$ in $\mathbb{P}^{2}$ is

$$
0 \rightarrow \mathscr{O}_{\mathbb{P}^{2}}(-5) \oplus \mathscr{O}_{\mathbb{P}^{2}}(-4) \rightarrow \mathscr{O}_{\mathbb{P}^{2}}(-3)^{3} \rightarrow \mathscr{T}_{X} \rightarrow 0 .
$$

By a theorem of L. Chiantini and F. Orecchia (see [CO]) we can conclude that there is a smooth, arithmetically Cohen-Macaulay curve $C$ in $\mathbb{P}^{3}$, one of whose hyperplane sections is $X$.

In fact, a closer look at the construction given in [CO] shows that if we choose three general linear forms $L_{1}, L_{2}, L_{3}$ in $k\left[X_{0}, X_{1}, X_{2}, X_{3}\right]$ and three general 
elements $a_{1}, a_{2}, a_{3}$ in $k$, then the maximal minors of the matrix

$$
\left(\begin{array}{ccc}
X_{1}-2 X_{0}+a_{1} X_{3} & X_{2}+a_{2} X_{3} & a_{3} X_{3} \\
\left(X_{2}-X_{0}\right)\left(X_{2}-2 X_{0}\right)+L_{1} X_{3} & L_{2} X_{3} & X_{1}\left(X_{1}-X_{0}\right)+L_{3} X_{3}
\end{array}\right)
$$

define a smooth, arithmetically Cohen-Macaulay curve $C$ such that $X$ is the intersection of $C$ and the hyperplane $\mathscr{V}\left(X_{3}\right)$.

We wonder which smooth, arithmetically Cohen-Macaulay curves have the property that every hyperplane section is a CB-scheme. Clearly smooth complete intersections do. What are some others?

\section{REFERENCES}

[BV] W. Bruns and U. Vetter, Determinantal rings, Lecture Notes in Math., vol. 1327, SpringerVerlag, Heidelberg, 1988.

[C] G. Castelnuovo, Sui multipli di una serie lineare di gruppi di punti appartenente ad una curva algebrica, Rend. Circ. Mat. Palermo 7 (1893), 89-110.

[CO] L. Chiantini and F. Orecchia, Plane sections of arithmetically normal curves in $\mathbb{P}^{3}$, Algebraic Curves and Projective Geometry, Proceedings, Trento 1988, Lecture Notes in Math., vol. 1389, Springer-Verlag, Heidelberg, 1989, pp. 32-42.

[D] E. D. Davis, Open problems, The Curves Seminar at Queen's, vol. III, Queen's Papers in Pure and Appl. Math. no. 67, Queen's University, Kingston, 1984, p. 8.

[DGM] E. D. Davis, A. V. Geramita, and P. Maroscia, Perfect homogeneous ideals: Dubreil's theorem revisited, Bull. Sci. Math. (2) 108 (1984), 143-185.

[DGO] E. D. Davis, A. V. Geramita, and F. Orecchia, Gorenstein algebras and the Cayley-Bacharach theorem, Proc. Amer. Math. Soc. 93 (1985), 593-597.

[G] W. Gröbner, Über irreduzible Ideale in kommutativen Ringen, Math. Ann. 110 (1934), 197-222.

[GM] A. V. Geramita and J. C. Migliore, Hyperplane sections of a smooth curve in $\mathbb{P}^{3}$, Comm. Algebra 17 (1989), 3129-3164.

[GMR] A. V. Geramita, P. Maroscia, and L. G. Roberts, The Hilbert function of a reduced $k$ algebra, J. London Math. Soc. (2) 28 (1983), 443-452.

[GN] A. Giovini and G. Niesi, CoCoA user's manual, v. 099b, Dipartimento di Matematica, Università di Genova, Genova, 1989.

[GW] S. Goto and K. Watanabe, On graded rings. I, J. Math. Soc. Japan 30 (1978), 179-213.

[H] J. Harris, The genus of space curves, Math. Ann. 249 (1980), 191-204.

[HE] J. Harris (with the collaboration of D. Eisenbud), Curves in projective space, Sém. de Mathématiques Supérieures, Université de Montreal, 1982.

[K1] M. Kreuzer, On 0-dimensional complete intersections, preprint, The Curves Seminar at Queen's, vol. VII, Queen's Papers in Pure and Appl. Math., no. 85, Queen's University, Kingston, 1990.

[K2] — Vektorbündel und der Satz von Cayley-Bacharach, Dissertation, Regensburger Mathematische Schriften 21, Universität Regensburg, 1989.

[KW] E. Kunz and R. Waldi, Regular differential forms, Contemporary Math., vol. 79, Amer. Math. Soc., Providence, R.I., 1988.

[O] F. Orecchia, Points in generic position and the conductor of curves with ordinary singularities, J. London Math. Soc. (2) 24 (1981), 85-96.

[Ra] J. Rathmann, The uniform position principle for curves in characteristic $p$, Math. Ann. 276 (1987), 565-579.

[Ro] L. Robbiano, Introduction to the theory of Gröbner bases, The Curves Seminar at Queen's, vol. V, Queen's Papers in Pure and Appl. Math., no. 80, Queen's University, Kingston, 1988, B1-B29. 
[So] A. Sodhi, On the conductor of points in $\mathbb{P}^{n}$, Dissertation, Queen's University, Kingston, 1987.

[St] R. Stanley, On the Hilbert function of a graded Cohen-Macaulay domain, preprint, Massachusetts Inst. of Technology, Cambridge, 1990.

Fakultat fur Mathematik, Universitat Regensburg, Postfach 397, D-W8400 RegensBURG, Federal RePUblic OF Germany

E-mail address: kreuzer@vax1.rs.uni-regensburg.dbp.de

Current address: A. V. Geramita, Department of Mathematics and Statistics, Queen's University, Kingston, Ontario, Canada K7L 3N6

E-maill address: geramita@qucdn.queensu.ca

Current address: L. Robbiano, Department of Mathematics, University of Genova, via L. B. Alberti 4, Genoa (16132), Italy

E-mail address: robbiano@igecuniv 